\title{
Imaging of brain tumors in children: the basics-a narrative review
}

\author{
Lucas Lopes Resende ${ }^{1}$, César Augusto Pinheiro Ferreira Alves ${ }^{2 \wedge}$ \\ ${ }^{1}$ Radiology Institute, University of São Paulo, São Paulo, Brazil; ${ }^{2}$ Department of Radiology, Division of Neuroradiology, Children's Hospital of \\ Philadelphia, Philadelphia, PA, USA \\ Contributions: (I) Conception and design: All authors; (II) Administrative support: All authors; (III) Provision of study materials or patients: All \\ authors; (IV) Collection and assembly of data: All authors; (V) Data analysis and interpretation: All authors; (VI) Manuscript writing: All authors; (VII) \\ Final approval of manuscript: All authors. \\ Correspondence to: César Augusto Pinheiro Ferreira Alves. Children's Hospital of Philadelphia, Department of Radiology, 3401 Civic Center Blvd, \\ Wood W225, Philadelphia, PA 19104, USA. Email: alvesc@email.chop.edu.
}

\begin{abstract}
Primary pediatric brain tumors comprise a broad group of neoplasm subtypes that can be categorized based on their histological and molecular features according to the 2016 World Health Organization (WHO) classification of central nervous system (CNS) tumors. The majority of the pediatric brain tumors demonstrate a singular preference for this age group and have a unique molecular profile. The separation of certain tumor entities, including different types of embryonal tumors, low-grade gliomas, and high-grade gliomas, may have a significant impact by guiding appropriate treatment for these children and potentially changing their outcomes. Currently, the focus of the imaging diagnostic studies is to follow the molecular updates, searching for potential imaging patterns that translate this information in molecular profile results, therefore helping the final diagnosis. Due to the high impact of accurate diagnosis in this context, the scientific community has presented extensive research on imaging pediatric tumors in recent years. This article summarizes the key characteristics of the imaging features of the most common primary childhood brain tumors, categorizing them according to the recent WHO classification update, which is based on each of their molecular profiles. The purpose of this review article is to familiarize radiologists with their key imaging features and thereby improve diagnostic accuracy.
\end{abstract}

Keywords: Pediatric brain tumors; neuroimaging; World Health Organization (WHO) 2016 classification; molecular profiles

Submitted Sep 06, 2020. Accepted for publication Mar 03, 2021.

doi: $10.21037 / \mathrm{tp}-20-285$

View this article at: http://dx.doi.org/10.21037/tp-20-285

\section{Introduction}

Central nervous system (CNS) tumors are the most common solid organ tumors in children and include nonmalignant and malignant neoplasms. In children (0-14 years age group) primary brain tumors had their highest incidence between 2012 and 2016, with a rate of 5.74 per 100,000 (1). Childhood CNS cancer was also the top reason for cancer mortality in children in 2009 (2).

Unlike neoplasms in other locations, primary brain tumors are not staged, but categorized according to the World Health Organization (WHO) 2016 Classification, which relies on molecular parameters in addition to histology to define their entities (3). Our goal is to offer an overview of these tumors, describing the imaging characteristics of the most common primary brain tumors in children based on the WHO classification update. We present the following article in accordance with the Narrative Review reporting checklist (http://dx.doi. org/10.21037/tp-20-285).

^ ORCID: 0000-0001-5877-9086. 


\section{The 2016 WHO classification of brain tumors with focus on the pediatric population}

In 2016 the WHO made significant changes to the classification of CNS tumors. This classification introduced a new framework for CNS tumor diagnoses in the molecular era by integrating molecular and genetic profiling into the diagnosis (3).

Some molecular profiles in pediatric neoplasms allow the distinction of certain entities from histologically similar adult counterparts. For example, the diffuse midline glioma H3 K27M-mutant previously included tumors termed as diffuse intrinsic pontine glioma (DIPG); now these have been classified as a new and single entity. Despite having a similarly poor outcome as glioblastomas do in adults, this specific glioma warrants a distinct category since it has different molecular features. The identification of this phenotypically and molecularly defined set of tumors provides a rationale for therapies directed against the effects of these mutations (4-6).

\section{Pediatric gliomas: overview}

Gliomas are the most common primary CNS tumors in the pediatric population and can be divided in two major groups: low-grade and high-grade gliomas. Low-grade gliomas are as common as malignant gliomas and embryonal tumors combined. They are mostly localized tumors (noninfiltrative tumors), and differ from $I D H$-mutant low-grade gliomas occurring in adults in that they rarely undergo malignant transformation, and show excellent overall survival under current treatment strategies $(7,8)$. Pediatric high-grade gliomas include malignant, diffuse, infiltrating astrocytic tumors such as anaplastic astrocytoma (WHO grade III) and glioblastoma (GBM; WHO grade IV). In 2016, the updated $\mathrm{WHO}$ criteria classified diffuse midline gliomas with $K 27 M$ histone mutations as $W H O$ grade $I V$, regardless of histology $(3,9)$, encompassing other midline gliomasincluding the most common, the DIPG, which behaves aggressively even when displaying lower-grade histology.

\section{Low-grade gliomas}

Low-grade gliomas are heterogeneous entities representing approximately $30 \%$ of all primary CNS tumors in childhood. They are typically slow-growing lesions classified as $W H O$ grade $I$ and II tumors $(8,10)$. Clinical presentation of childhood gliomas is heterogeneous and is dependent on the location of the lesion. Cortical tumors typically manifest with focal neurological deficits, while brainstem gliomas might present with hydrocephalus and headache, fatigue, ataxia, visual changes, facial weakness, and gagging. Low-grade gliomas typically take protracted clinical courses, while high-grade tumors tend to have a more acute onset with rapid progression of symptoms (11).

Low-grade gliomas are a group that describes tumors of primarily glial histology (including astrocytic and/ or oligodendroglial). The different histologic entities are distinguished as many low-grade gliomas; however, there are some cases of overlapping morphology, such as pleomorphic xanthoastrocytoma and ganglioglioma, dysembryoplastic neuroepithelial tumor (DNET), and oligodendroglioma (12). Low-grade gliomas may also overlap morphologically with entities more commonly found in adults, but there are marked differences between the two on the molecular and genetic levels (11).

Management of low-grade gliomas is usually related to surgical resection; complete resection is the most favorable predictor of patient outcome. In the past, complete surgical resection, histological diagnosis, and age were used to determine prognosis. Currently, the molecular data of lowgrade gliomas supplement the stratification of these tumors and their prognosis (12). Here we describe demographics, clinical features, and the molecular profile from the most common pediatric low-grade gliomas. The authors also provide a summary table that includes an overview of the imaging appearances of the most common low-grade gliomas along with their most frequent locations in the brain, their genetic features, and expected outcome (Table 1).

\section{Pilocytic astrocytoma}

Pilocytic astrocytoma is the most common brain glioma in children, approximately $16 \%$ of brain neoplasms, and is designated as WHO I tumors of the brain (13). They are a distinct histologic type of glioma that have a slow growth rate and may even spontaneously regress (14). They can be found in any region of the CNS, including the supratentorial or infratentorial fossa, particularly affecting the optic pathway, and also the spinal cord $(15,16)$.

The most common location of pilocytic astrocytomas is the cerebellum. Depending on the size and location of the tumor it may be associated with mass effect, causing hydrocephalus and signs of elevated intracranial pressure, potential clinical features at the onset. 
Table 1 Summary table of low grade gliomas

\begin{tabular}{|c|c|c|c|c|c|}
\hline *Neoplasm & Distinct imaging features & Locations & $\begin{array}{l}\text { Histology and } \\
\text { molecular }\end{array}$ & Grade (WHO) & Outcome \\
\hline $\begin{array}{l}\text { Pilocytic } \\
\text { astrocytoma }\end{array}$ & $\begin{array}{l}\text { Cystic lesion with a mural } \\
\text { enhancing nodule (classic } \\
\text { for cerebellar location). More } \\
\text { infiltrative lesions without } \\
\text { cystic component may be } \\
\text { seen in the optic pathway } \\
\text { and tegmental plate }\end{array}$ & $\begin{array}{l}\text { Cerebellum } \\
\text { (hemispheres and } \\
\text { vermis). Optic } \\
\text { pathway (NF1). } \\
\text { Tegmental plate } \\
\text { (causing aqueduct } \\
\text { stenosis). Other } \\
\text { locations }\end{array}$ & $\begin{array}{l}\text { GFAP+, S100+, } \\
\text { BRAF alterations } \\
\text { (variable frequency } \\
\text { according to the } \\
\text { location and age } \\
\text { of onset) }\end{array}$ & I & $\begin{array}{l}\text { Favorable } \\
\text { prognosis }\end{array}$ \\
\hline $\begin{array}{l}\text { Pilomyxoid } \\
\text { astrocytoma }\end{array}$ & $\begin{array}{l}\text { Predominantly solid tumors. } \\
\text { Cystic components tend to } \\
\text { be located externally to the } \\
\text { core of the tumor }\end{array}$ & $\begin{array}{l}\text { Suprasellar region } \\
\text { extending to the } \\
\text { temporal lobes ("H } \\
\text { shape") }\end{array}$ & $\begin{array}{l}\text { GFAP+, S100+, } \\
50 \% \text { BRAF } \\
\text { alterations }\end{array}$ & Not determined & $\begin{array}{l}\text { Favorable } \\
\text { prognosis }\end{array}$ \\
\hline $\begin{array}{l}\text { Pleomorphic } \\
\text { xanthoastrocytoma }\end{array}$ & $\begin{array}{l}\text { Solid-cystic cortical } \\
\text { tumor extending to the } \\
\text { leptomeninges with vivid } \\
\text { contrast enhancement. } \\
\text { Scalloping of the overlying } \\
\text { bone and reactive dural } \\
\text { enhancement }\end{array}$ & $\begin{array}{l}\text { Predilection for the } \\
\text { temporal lobe }\end{array}$ & $\begin{array}{l}\mathrm{GFAP}+, \mathrm{S} 100+ \\
\text { BRAF alterations }\end{array}$ & II & $\begin{array}{l}\text { Favorable } \\
\text { prognosis. } \\
\text { However, local } \\
\text { recurrence } \\
\text { and malignant } \\
\text { transformation } \\
\text { may occur }\end{array}$ \\
\hline
\end{tabular}

*, low grade gliomas are more often hypointense on T1WI and hyperintense on T2WI without restricted DWI. **, possible feature indicating IDH mutation. NF1, Neurofibromatosis type 1.

\section{Molecular features}

Pilocytic astrocytomas frequently have $B R A F$ alterations, a molecular feature that varies its frequency according to the tumor location, more often noted in the cerebellar location, and usually do not present IDH and TP53 mutations, as do other pediatric low-grade gliomas (17).

\section{Imaging features}

The typical imaging pattern is a large cystic lesion with a mural nodule. On computed tomography (CT) studies there is an isodense nodule that may present contrast enhancement, surrounded by a hypodense cyst, more frequently located in one of the cerebellar hemispheres. On MRI the nodule is iso- or hypointense to gray matter on T1-weighted images (T1WI) and hyperintense on T2weighted images (T2WI). The signal intensity of the cystic component is often similar to the cerebro spinal fluid (CSF). Other typical imaging findings observed on MRI are absence of restricted water movement, depicted on diffusion weighted imaging (DWI); and presence of avid gadolinium enhancement of the solid portion, or even in the cystic walls of the tumor on T1-weighted imaging (post- 

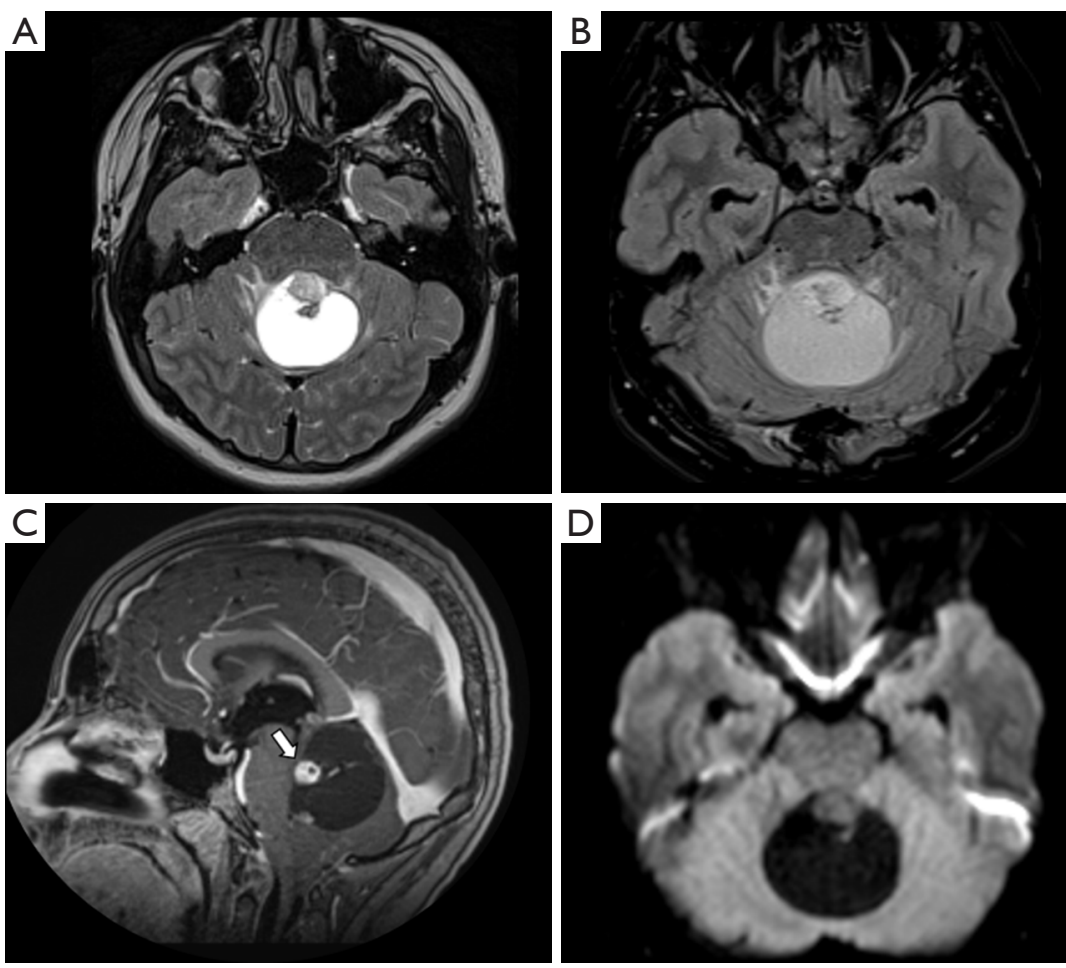

Figure 1 Male, 13 years old, diagnosis of pilocytic astrocytoma KIAA1549-BRAF fusion. Brain MRI: Axial T2WI, axial FLAIR, sagittal T1W post-contrast, and DWI (A,B,C,D) show a solid-cystic tumor located in the superior aspect of the vermis, hyperintense on T2WI, and presenting a large cystic component with a mural avid enhancing nodule (arrow, C). No diffusion restriction was observed (D). DWI, diffusion weighted imaging; FLAIR, fluid-attenuated inversion recovery; T1W, T1-weighted; T2WI, T2-weighted image.

contrast T1WI) (Figure 1). About 5\% to $10 \%$ of pilocytic astrocytomas demonstrate leptomeningeal spread; however, this does not change their 2016 WHO categorization. It is important to highlight that pilocytic astrocytomas may present with other imaging characteristics such as multiple cysts, necrosis, hemorrhage, calcification (Figure 2), or even as a completely solid tumor, which may pose a challenge for radiologists in diagnosis $(18,19)$.

Moreover, although advanced imaging techniques are out of the scope of this paper, it is important to highlight that some particular imaging features suggesting aggressive behavior from these techniques [such as Arterial Spin Label (ASL) showing increased perfusion of the tumor and $1 \mathrm{H}-\mathrm{MRS}$ showing elevated lipid-lactate peaks in $1.3 \mathrm{ppm}]$ can also be noted in localized/low-grade tumors, particularly in pilocytic astrocytomas, mimicking the imaging features of high-grade tumors (Figure 3). This should be kept in mind during image interpretation (20).

The second most common location is the optic pathway, particularly in neurofibromatosis I (NF1) patients. Optic pathway gliomas can be confined to the optic nerve, or may extend to the optic chiasm, optic radiations, and hypothalamus. When the tumor is restricted to the optic nerve, it presents itself as an enlarged optic nerve with or without enhancement, with minimal or absent cystic components. Larger tumors involving the chiasm and hypothalamus are usually present as heterogeneous nodules/ masses with cystic and solid components and infiltrative features $(19,21)$.

The main differential diagnoses include other low-grade gliomas, particularly those located in the posterior fossa with solid-cystic appearance, such as hemangioblastomas (more frequent in adults); embryonal tumors [medulloblastomas and atypical teratoid rhabdoid tumors (AT/RT), both more often presenting with restricted diffusivity on DWI sequence]; ependymomas (more frequently observed with a "plastic" appearance, hemorrhage, and calcification); and inflammatory infectious processes such as an abscess.

\section{Pilomyxoid astrocytoma}

Pilomyxoid astrocytoma grading has changed according 

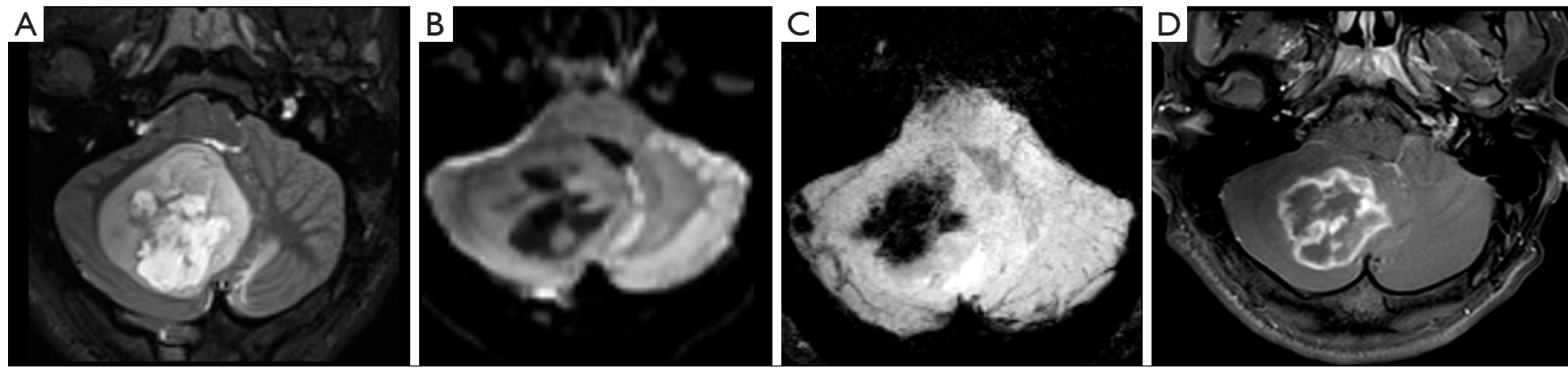

Figure 2 Male, 6 years old, diagnosis of pilocytic astrocytoma KIAA1549-BRAF fusion. Brain MRI: Axial T2WI, DWI, SWI, and T1W post-contrast (A,B,C,D) show a solid-cystic tumor located in the right cerebellar hemisphere and vermis, with mass effect, and heterogeneous hyperintense signal on T2WI (A). The tumor shows no diffusion restriction (B), a central component of hemorrhage characterized by a hypointense signal on SWI (C), confirmed on a CT study (not shown), and necrosis delineated by areas of enhancement (D). DWI, diffusion weighted imaging; SWI, susceptibility weighted imaging; T1W, T1-weighted; T2WI, T2-weighted image.

to the WHO 2016 classification. In the WHO 2007 classification, pilomyxoid astrocytomas were designated as WHO grade II; however, due to the many histological and genetic similarities between pilomyxoid astrocytomas and suprasellar pilocytic astrocytomas, the previous grading of pilomyxoid astrocytomas has been removed and is now included as a potential variant of pilocytic astrocytomas (3). Features that support this re-designation include the younger ages of patients with pilomyxoid astrocytoma compared with those of patients with pilocytic astrocytoma, and the selective involvement of the suprasellar regions (22). Despite the new WHO "classification", results from previous studies have shown that pilomyxoid astrocytomas follow a more aggressive course than pilocytic astrocytomas, and radiologic evidence shows more frequent CSF dissemination in pilomyxoid astrocytomas when compared with pilocytic astrocytomas (22-24).

\section{Molecular features}

$B R A F$ fusions are the most relevant molecular marker for pilomyxoid astrocytomas, present in around $50 \%$ of all these tumors. The fact that this molecular feature is less frequently seen in these tumors than the classical pilocytic astrocytoma may support the possibility that pilomyxoid astrocytomas are just less mature forms of pilocytic astrocytomas (25).

\section{Imaging features}

Pilomyxoid astrocytomas are predominantly solid tumors. Their cystic components, when present, tend to be located externally to the core of the tumor. The tumor may extend into the temporal lobes (26), giving an "H shape" imaging feature in the suprasellar region, a relatively characteristic finding observed in coronal planes. On MRI, these tumors are more frequently hypointense on T1WI and hyperintense on T2WI. They show homogeneous or heterogeneous post-contrast T1WI enhancement, and areas of hemorrhage may be observed in up to $25 \%$ of them (Figure 4) (24).

The differential diagnoses may include other tumors centered on the hypothalamic-chiasmatic region, including other low-grade gliomas such as pilocytic astrocytoma; craniopharyngiomas, which more frequently present with cystic/multicystic components and iso- to hyperintense signal on T1W1 due to high protein concentration; germ cell tumors (GCTs), particularly non-pure germinomas due to the more heterogeneous appearance and large size; and macroadenomas, which are not often observed in young children.

\section{Diffuse astrocytoma}

Diffuse astrocytomas are less common than pilocytic astrocytomas in children. Designated as a WHO II brain tumor, this type is also referred to as low-grade infiltrative astrocytoma. The term "diffuse" means that there is no histologic border between the tumor and normal brain tissue, even if the border appears well-delineated on imaging. These tumors can be located anywhere in the encephalon, but are more frequently observed in the supratentorial compartment.

\section{Molecular features}

$I D H$ mutation status is the most relevant molecular 

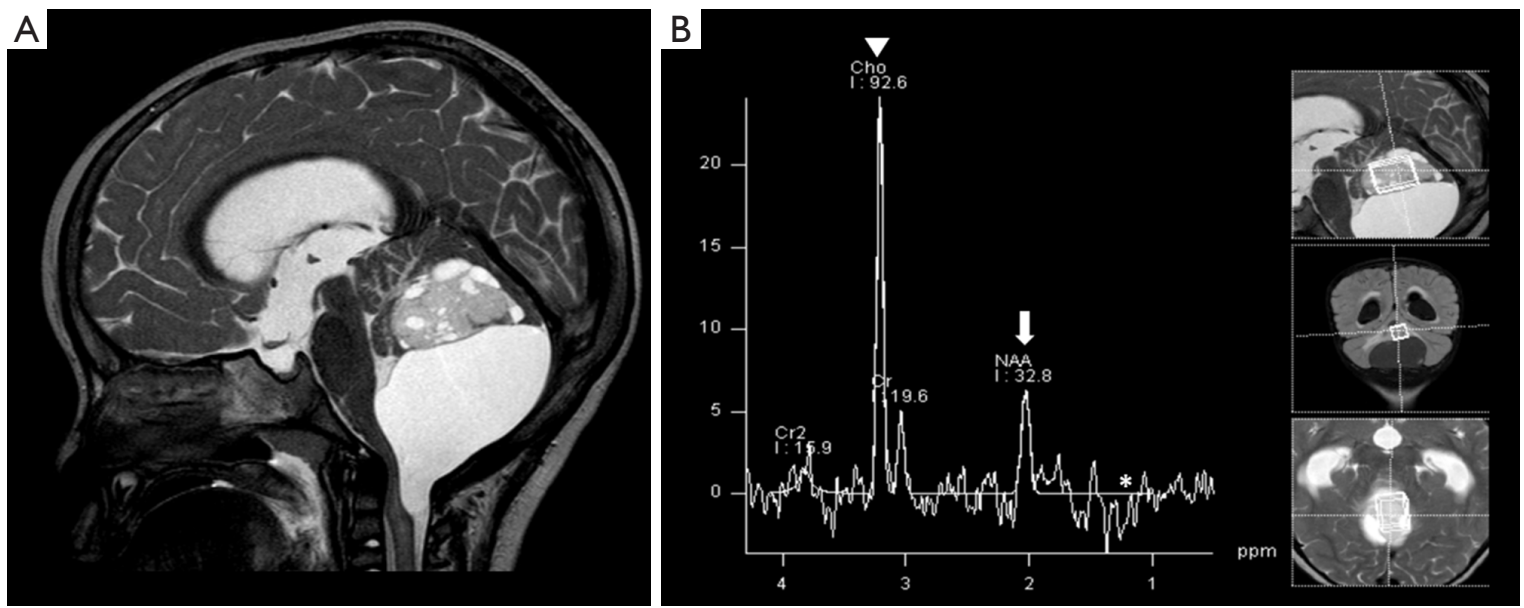

Figure 3 Solid cystic mass in the posterior fossa was confirmed as pilocytic astrocytoma. Brain MRI: Sagittal T2WI, and 1H MRS (A,B). Reduced NAA (arrow), increased choline (arrowhead), and possible mild lactate (asterisk) peaks were observed (B). T2WI, T2-weighted image; MRS, magnetic resonance spectroscopy; NAA, N-acetylaspartate.
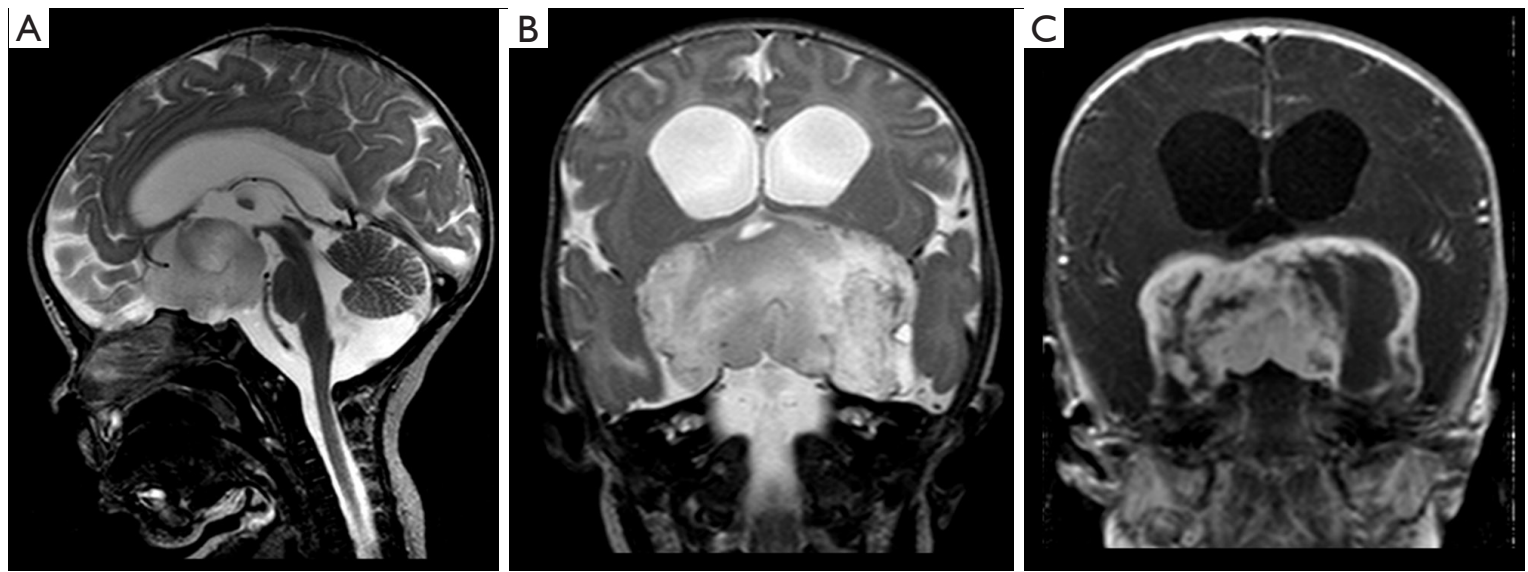

Figure 4 Male, 2 years old, diagnosis of pilomyxoid astrocytoma. Brain MRI: Sagittal T2WI, coronal T2WI and T1W post-contrast $(\mathrm{A}, \mathrm{B}, \mathrm{C})$ show a suprasellar infiltrative mass hyperintense on T2WI, with cystic components peripherally distributed, and heterogeneous post-contrast T1WI enhancement (C), which extends into the temporal lobes, giving an "H shape" on coronal imaging (B,C). T1W, T1weighted; T2WI, T2-weighted image.

marker because it is related to prognosis. Currently diffuse astrocytomas are classified into two molecular groups: $I D H$ mutant or $I D H$ wild type. If $I D H$ status is not available, the tumor will be classified as IDH NOS (not otherwise specified). IDH mutant tumors also need to be tested for $1 \mathrm{p} 19 \mathrm{q}$ status, since $I D H$ mutant and $1 \mathrm{p} 19 \mathrm{q}$ co-deleted tumors are referred to as oligodendrogliomas and have different prognosis and treatments $(11,12,27)$.

\section{Imaging features}

On MRI, diffuse astrocytomas are usually depicted as a mass-like signal abnormality, often appearing without a distinct tumor border, and located in the juxtacortical white matter. The cortex may or may not be affected and a mild mass effect is often present, effacing the underlying cortical sulci. In some cases, microcystics may be visible along with the tumor; this feature is a hallmark of infiltrative astrocytomas, albeit uncommon. This tumor often does not show significant enhancement on postcontrast T1WI sequences. If enhancement is observed, it may indicate progression or a higher-grade tumor. A potential imaging sign that may suggest this diagnosis of a low-grade diffuse 
astrocytoma $I D H$ mutated is the T2/fluid-attenuated inversion recovery (FLAIR) mismatch, although it is not always present (28).

Differential diagnoses include high-grade gliomas, but those tend to present with areas of necrosis and more avid enhancement; inflammatory/infection lesions such as abscesses, and less frequently vascular abnormalities $(27,29,30)$.

\section{Pleomorphic Xanthoastrocytoma}

Pleomorphic xanthoastrocytoma is a rare type of low-grade astrocytoma (WHO grade II) located in the supratentorial compartment typically observed in patients aged 10 to 30 years who have temporal lobe epilepsy.

\section{Molecular features}

Over two-thirds of pleomorphic xanthoastrocytomas harbor mutations in $B R A F$, which conveys a more favorable natural history for this tumor (31). Grade subclassifications based on the presence of wild or mutated $B R A F$ have been considered for pleomorphic xanthoastrocytoma since this molecular feature may predict the outcome.

\section{Imaging features}

The most common presentation is a solid cystic cortical tumor with vivid contrast enhancement on postcontrast T1WI sequences. Findings associated with slow growth may appear, such as scalloping of the overlying bone and/or absence of white matter edema (Figure 5). A reactive dural enhancement may also be visible. Calcifications are not common. Differential diagnoses include other juxtacortical/ cortical tumors such as DNET, gangliogliomas, oligodendrogliomas, or even cystic meningiomas $(32,33)$.

\section{Angiocentric glioma}

Angiocentric glioma is a rare, slow-growing infiltrative cortical or juxtacortical low-grade neoplasm found in young patients with refractory epilepsy, designated as a WHO I brain tumor that often presents with immunophenotype similarities to ependymomas. They may be associated with cortical dysplasia, as well as DNETs (34). Angiocentric gliomas are most commonly located in the supratentorial compartment, particularly in the frontoparietal and temporal lobes as well as the hippocampal region (35).

\section{Molecular features}

Previous studies noted that a myeloblastosis quaking (MYB-
QKI) gene rearrangement was a distinct feature observed in the majority of angiocentric gliomas, while IDH1 mutation was not noted $(36,37)$. This molecular profile may help the differential diagnosis of angiocentric gliomas from other gliomas and IDH1-mutated glioblastomas (35).

\section{Imaging features}

On imaging, angiocentric gliomas appear as well-delineated, non-contrast-enhancing cortical or subcortical (graywhite matter junction) tumors, which tend to expand the affected gyri. This tumor is more frequently described as hypointense on T1WI and hyperintense on T2WI (38); however, a hyperintense rim on T1WI may be seen and can help to narrow the differential diagnosis (39). Calcification is not a hallmark, but may be found in some cases $(38,40)$. Differential diagnoses include other juxtacortical/cortical tumors such as pleomorphic xanthoastrocytoma, DNET and gangliogliomas.

\section{Astroblastoma}

Astroblastomas are rare neoplasms of uncertain origin more often observed in the brain hemispheres of patients aged between 10 and 30 years. There is not yet a WHO grade for this entity. Its biological behavior varies from a relatively indolent tumor to an aggressive anaplastic astroblastoma.

\section{Molecular features}

This tumor has a heterogeneous molecular profile without a particular genetic biomarker. The extensive variability of molecular features for these tumors is probably linked to their clinical unpredictability (41).

\section{Imaging features}

Astroblastomas tend to present as supratentorial solidcystic masses, peripherally located with mild or no adjacent vasogenic edema. Heterogeneous areas of gadolinium enhancement on postcontrast T1WI sequences, associated with punctate calcifications on T2WI and susceptibility sequences, and a bubbly and multicystic appearance are the imaging hallmarks of this tumor $(42,43)$. Differential diagnoses also include other juxtacortical/cortical tumors such as pleomorphic xanthoastrocytoma, DNET and gangliogliomas.

\section{Pediatric high-grade gliomas}

High-grade gliomas are the most prevalent primary CNS 

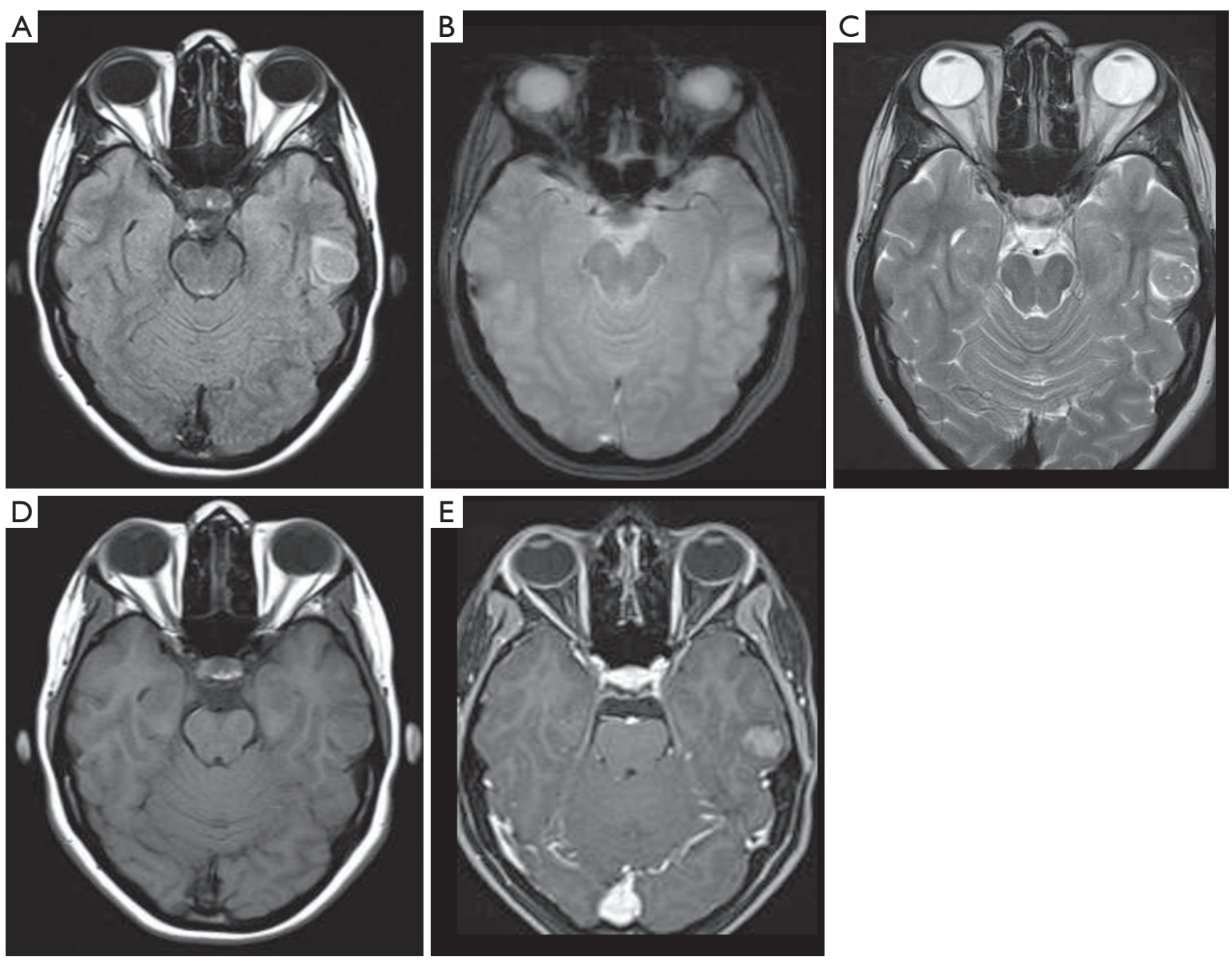

Figure 5 Female, 14 years old, diagnosis of pleomorphic xanthoastrocytoma. Brain MRI: Axial FLAIR, T2 Gradient-echo, T2WI, T1WI, and T1W post-contrast (A,B,C,D,E) show a cortical and juxtacortical well-circumscribed lesion located in the left temporal lobe, hypointense to the cortex on FLAIR/T2WI (A,C), without signs of calcification or hemorrhage on T2 Gradient-echo (B), and post-contrast T1WI homogeneous enhancement (E). FLAIR, fluid-attenuated inversion recovery; T1WI, T1-weighted image; T2WI, T2-weighted image.

tumors in adults, but occur as only $8-12 \%$ of all primary CNS tumors in children (44). Moreover, high-grade gliomas' characteristics differ between these two populations in terms of molecular, genetic, and biologic data (45). These tumors are classified by the WHO as either grade III or $I V$, the most common histologies being the anaplastic astrocytoma (WHO grade III) and the glioblastoma (WHO grade $I V)$. In terms of molecular features these tumors are classified as $I D H$-wild type and $I D H$ 1/2-mutated entities (3). Notably, the critical distinction that best describes the diffuse infiltrating gliomas in children is the identification of somatic histone mutations, which are not found in adults (46). Mutations in the genes encoding the H3.3 (H3F3A) and H3.1 (HIST1H3B, HIST1H3C) histone variants result in amino acid substitutions and yield the following tumors: H3.3 G34 localized in the cerebral hemispheres, H3.3 K27M found in the midline structures (thalamus, brainstem, cerebellum, and spine), and H3.1 $K 27 M$ limited to the pons (47-50). The authors also provide a summary table that includes an overview of the imaging appearance of the most common high-grade gliomas along with their most frequent location in the brain, genetic features, and expected outcome (Table 2).

\section{Diffuse midline glioma, H3K27M-mutant}

Diffuse midline glioma (DMG) is a devastating tumor that mainly affects the pediatric population, with a high incidence among children around 5 to 11 years old. This new entity was recently included in the 2016 WHO 
Table 2 Summary table of pediatric high-grade gliomas

\begin{tabular}{|c|c|c|c|c|c|}
\hline *Neoplasm & Distinct imaging features & Locations & $\begin{array}{l}\text { Histology and } \\
\text { molecular }\end{array}$ & Grade $(\mathrm{WHO})$ & Outcome \\
\hline $\begin{array}{l}\text { **Diffuse Midline Glioma } \\
\text { (DMG), H3K27M }\end{array}$ & $\begin{array}{l}\text { Midline tumor with an } \\
\text { infiltrative pattern. When } \\
\text { arising from pons causes } \\
\text { a diffuse enlargement of } \\
\text { this structure } \\
\text { "engulfing" anteriorly } \\
\text { the basilar artery. Subtle } \\
\text { enhancement (linear or } \\
\text { punctate) may be seen. } \\
\text { Areas of necrosis only } \\
\text { in the late stage or after } \\
\text { treatment }\end{array}$ & $\begin{array}{l}\text { Brainstem, } \\
\text { particularly the } \\
\text { pons, but may } \\
\text { arise/infiltrate the } \\
\text { basal ganglia, } \\
\text { thalami, all parts } \\
\text { of the brainstem } \\
\text { and spinal cord }\end{array}$ & $\begin{array}{l}\text { GFAP variable, } \\
\mathrm{S} 100+, \mathrm{H} 3-\mathrm{K} 27 \mathrm{M}\end{array}$ & IV & Poor outcome \\
\hline
\end{tabular}

*, High grade gliomas are more often infiltrative hypointense on T1WI and hyperintense on T2WI with variable components of restriction on DWI; **, DMG with bithalamic involvement may lack H3 mutation but present with EGFR mutations which may confer selective vulnerability to EGFR kinase inhibitors.

Classification of Tumors, and is defined by distinct histologic and molecular features. DMG represents a grouping of several previously individualized neoplasms, including the DIPG (3).

Regarding the distribution of these tumors, DMG are most often observed in the pons, but can also be observed in other locations of the brainstem (midbrain and medulla) and in other anatomic structures such as thalamus, spinal cord, and cerebellum (51). Despite the overall poor outcome, survival differences are observed according to the anatomic distribution of these brainstem tumors and to the age of onset-neoplasms arising outside of the pons; in older children, they are apparently linked to a slightly better outcome (51). Extension of the tumor throughout the cerebellar peduncles may also be observed and it is associated with a worse outcome (52).

\section{Molecular features}

The rationale for the broad incorporation of DMGs in the latest classification relies on the fact that the majority of these gliomas have a similar molecular profile (H3K27M mutation) and a dismal prognosis, with a survival rate of less than $5 \%$ to $10 \%$ at 2 years following diagnosis. There is a small percentage of DMG tumors with a different molecular profile, but of these the DMG H3-K27M appear to represent the poorest prognosis (53). Moreover, intrinsic variations of $\mathrm{H} 3$ protein in tumors with the lysine 27 mutation may also be observed, and these variations have been associated with differences in survival. The overall survival in $\mathrm{H} 3.3$ protein tail is considered worse compared with H3.1-mutated subgroups (54).

\section{Imaging features}

The imaging features may vary according to the location in which the tumor arises (55). Tumors in the pons, most frequent ones, are more often observed in its ventral part (basilar pons), with an infiltrative pattern causing a diffuse enlargement of the pons, displacing and engulfing anteriorly the basilar artery (Figure 6) (55). Although rare, the tumor can present with exophytic features, and be located in the tegmentum of the pons. However, this dorsal and exofitic imaging presentation favors low-grade gliomas, particularly pilocytic astrocytomas.

Presence of necrosis is not expected in the first stages of the disease or before treatment. The tumor often has a variable T2WI signal intensity, not necessarily representing 

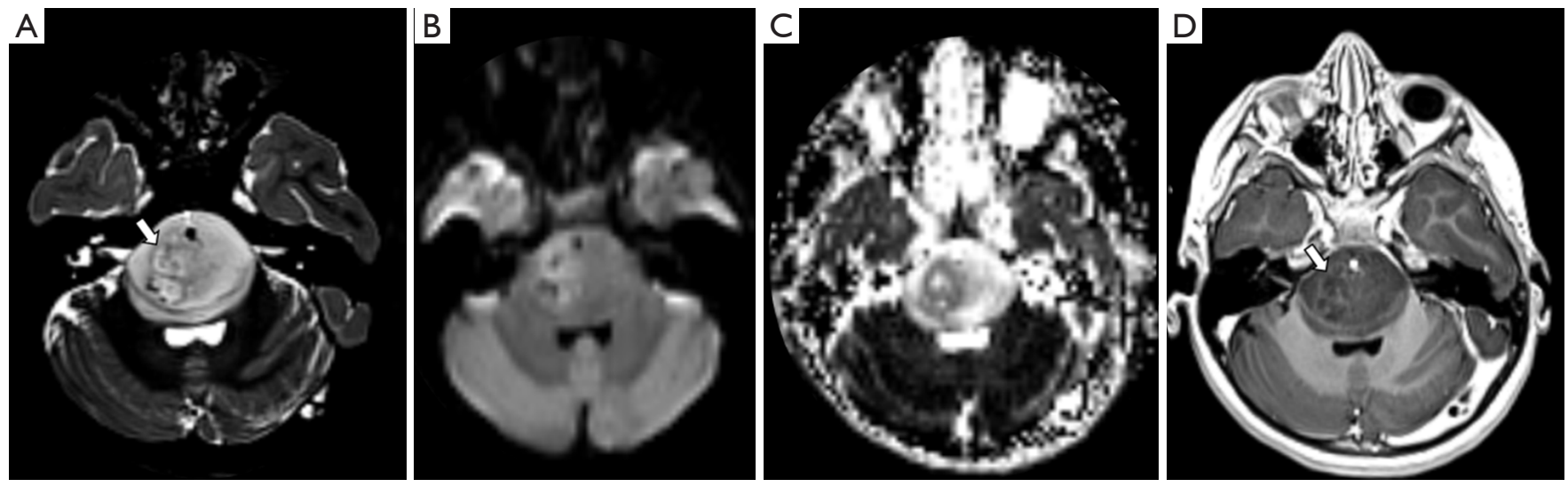

Figure 6 Female, 3 years old, diagnosis of diffuse midline glioma, H3K27M-mutant. Brain MRI: T2WI, DWI, ADC map and T1WI postcontrast (A,B,C,D) show an infiltrative mass in the anterior aspect of the pons, causing a diffuse enlargement of the pons (A), displacing and engulfing the basilar artery anteriorly, with areas of restricted diffusion (B,C) and mild contrast enhancement delineating areas of necrosis (arrows, A and D). ADC, apparent diffusion coefficient; DWI, diffusion weighted imaging; T1WI, T1-weighted image; T2WI, T2-weighted image.

areas of liquefaction or necrosis. Areas of enhancement are often subtle, with a mild linear or punctate form (52), and may vary according to the location of these tumors; pontine gliomas tend to enhance more frequently than the thalamic ones at a rate of $67 \%$ versus $50 \%$, respectively (55).

Components of DWI restriction are also variable in DMG, ranging more frequently between normal and mildly restricted. Although no clear statistically significant difference in diffusion characteristics is described among the different molecular profiles of DMG, gliomas with lower diffusivity correspond to a lower outcome, with death occurring around 1 year after diagnosis (53).

Follow-up studies often demonstrate extensive craniocaudal infiltration of the tumor, including involvement of the peduncles, diencephalon, and spinal cord. Presence of leptomeningeal spread is also frequently observed throughout the course of the disease (3). Differential diagnoses include low-grade gliomas arising in the midline; however, these tumors tend to be present with a well delineated margin, less mass effect, not engulfing anteriorly the basilar artery, and with more avid enhancement on postcontrast T1WI sequences when compared to DMG.

\section{Other high-grade pediatric gliomas}

Histone H3.3 G34 mutant gliomas are rare tumors in adolescents and young adults, and tend to grow in the forebrain hemispheres. Although this subtype is not formally identified as a distinct entity in the WHO classification 2016 (3), novel publications imply that H3.3 G34 mutant gliomas represent a separate tumor subgroup due to their clinical data, molecular biology, and some histologic features $(56,57)$. In addition, this subgroup shows a higher level of $M G M T$ methylation than do $K 27 M$ mutations, and carries frequent TP53 (88\%) and ATRX (95\%) alterations (58). Although classified as glioblastoma $I D H$ wild type, some of them lack common histological features of glioblastoma, such as microvascular proliferation and/or necrosis. These findings are reflected in the neuroimaging investigation, with some cases meeting criteria only for low-grade gliomas (e.g., absence of contrast enhancement and necrosis) (59). Their MRI features are heterogeneous, but more frequently described as infiltrative hemispheric tumors. Variable components of cysts, hemorrhage, and calcifications, along with areas of restricted diffusion and necrosis, may be found.

Beyond the subclassification of histone $H 3$ mutations, more than $50 \%$ of all pediatric patients with diffusely infiltrating gliomas can not be characterized in this category. Particular attention must be given to bithalamic gliomas, which, contrarily to the unilateral ones, lack $\mathrm{H} 3$ mutation, but present with EGFR mutations which may confer selective vulnerability to EGFR kinase inhibitors (60).

Few children $(<5 \%)$ present with mutations in the $I D H 1 / 2$ genes, which are associated with global hypermethylation ("G-CIMP") $(61,62)$. Imaging features that are associated with gliomas that present mutations in the $I D H 1$ and $I D H 2$ are the D-2-hydroxyglutarate (2-HG) in $2.25 \mathrm{ppm}$ in $1 H-M R S$ and the FLAIR-T2 "mismatch" $(30,57,63,64)$. 
Moreover, a larger pediatric cohort (5-10\%) demonstrated activated $B R A F$ V600E mutation (65), represented by predominantly cortical tumors, which have histological and epigenetic similarities to pleomorphic xanthoastrocytoma (4), and also have a better prognosis. Ultimately, the remaining tumors constitute a heterogeneous group, which are poorly defined with respect to their different molecular and clinicopathological hallmarks.

\section{Neuronal and mixed neuronal-glial tumors}

Neuronal and mixed neuronal-glial neoplasms are uncommon CNS tumors, more frequently categorized as $W H O$ grade I, and mainly observed in children and young adults.

The most common clinical feature associated with these tumors is epilepsy (refractory seizures) and the most common locations include the floor of the third ventricle, the temporal lobe, cerebellum, parieto-occipital region, and frontal lobe. Neuronal migration abnormalities in particular cortical dysplasias (focal cortical dysplasia - FCD type II), may coexist with glioneuronal tumors. As a consequence, imaging features of cortical dysplasia such as blurring of the cortical and juxtacortical junction, cortical thickness, and T2WI white matter changes (i.e., white matter transmantle sign), may overlap with the imaging features of these tumors (66). The authors provide a summary table that includes an overview of the imaging appearance of the most common neuroglial tumors, along with their most frequent location in the brain, genetic features, and expected outcome (Table 3).

\section{Ganglioglioma}

Gangliogliomas are the most frequent neoplasms among neuronal and mixed-glial tumors, designated as WHO I brain tumors, accounting for up to $10 \%$ of primary cerebral tumors in children. Approximately $70 \%$ of gangliogliomas occur in the temporal lobes, slow-growing over time. Similar to other neuroglial tumors, their most common clinical presentation in children is refractory epilepsy.

\section{Molecular features}

These tumors when presented as WHO grade I, a more common form, are often associated with BRAF V600E mutation, and $I D H$ status is usually negative. Less frequently, gangliogliomas may show an aggressive behavior (fewer than $10 \%$ ), presenting anaplastic areas, BTERT, $A T R X$ or histone genes mutation and are classified as a
WHO grade III tumor $(3,17,67,68)$.

\section{Imaging features}

Gangliogliomas are observed as a cystic lesion with a mural nodule in approximately $40 \%$ of diagnosed cases. Calcification has been found in about $30 \%$ of cases. Gangliogliomas may cause scalloped pressure erosion of the overlying calvaria due to their slow-growing nature. On MRI, a well-defined cystic mass with a solid mural nodule is typically observed. Enhancement of the solid portion is variable, ranging from none to intense. A mild mass effect and surrounding vasogenic edema are also commonly seen $(67,69)$ (Figure 7). Infiltrating appearance is uncommon and when present is usually related to a higher-grade tumor (70). Differential diagnoses include other low-grade gliomas with solid-cystic appearance, cortical/juxtacortical located, and clinically associated with seizures, and focal cortical dysplasias, particularly FCD type II that may or may not be associated with gangliogliomas.

\section{Lbermitte-duclos disease}

Dysplastic cerebellar gangliocytoma (Lhermitte-Duclos disease) is a rare form of neuronal-glial tumor that distorts the normal cerebellar laminar cytoarchitecture. Its pathogenesis remains unclear. Even though it may not be a neoplasia, it is considered a $W H O$ grade I tumor with slow or absent growth even after years and good outcome.

\section{Molecular features}

A PTEN mutation may be found when the tumor appears in the context of COWDEN syndrome (autosomal dominant inherited disorder presented with multiple hamartomas and increased risk for different types of neoplasms).

\section{Imaging features}

On MRI, the lesion appears as a nonenhancing mass affecting one, or less frequently, both cerebellar hemispheres, causing a mild mass effect, and has a typical striated pattern described as "corduroy/laminated" appearance (Figure 8) $(3,71,72)$. The main differential diagnosis includes some cerebellar malformative features with a pseudotumoral appearance, and other low-grade gliomas arising from the cerebellar hemispheres.

\section{Diffuse leptomeningeal glioneuronal tumor}

Diffuse leptomeningeal glioneuronal tumors are a rare, 
Table 3 Summary table of neuronal-glial tumors

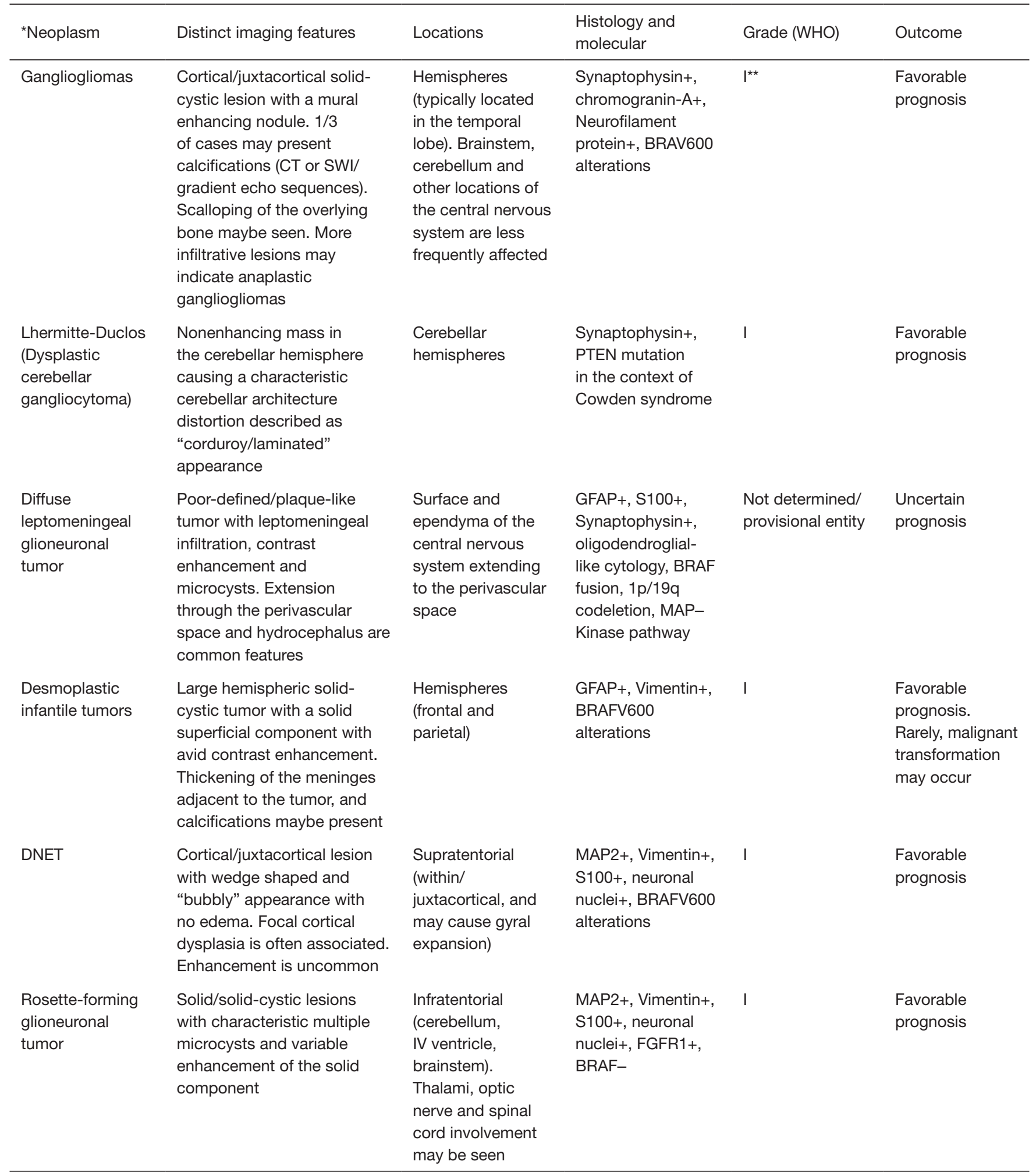

*, Neuronal-glial tumors are more often solid-cystic cortical or juxtacortical lesions hypointense on T1WI and hyperintense on T2WI, without restricted DWI. Overlapping imaging features of cortical dysplasia and microcysts maybe seen. **, Anaplastic Gangliogliomas are less frequent Grade III tumors with a poor prognosis. DNET, dysembryoplastic neuroepithelial tumor. 

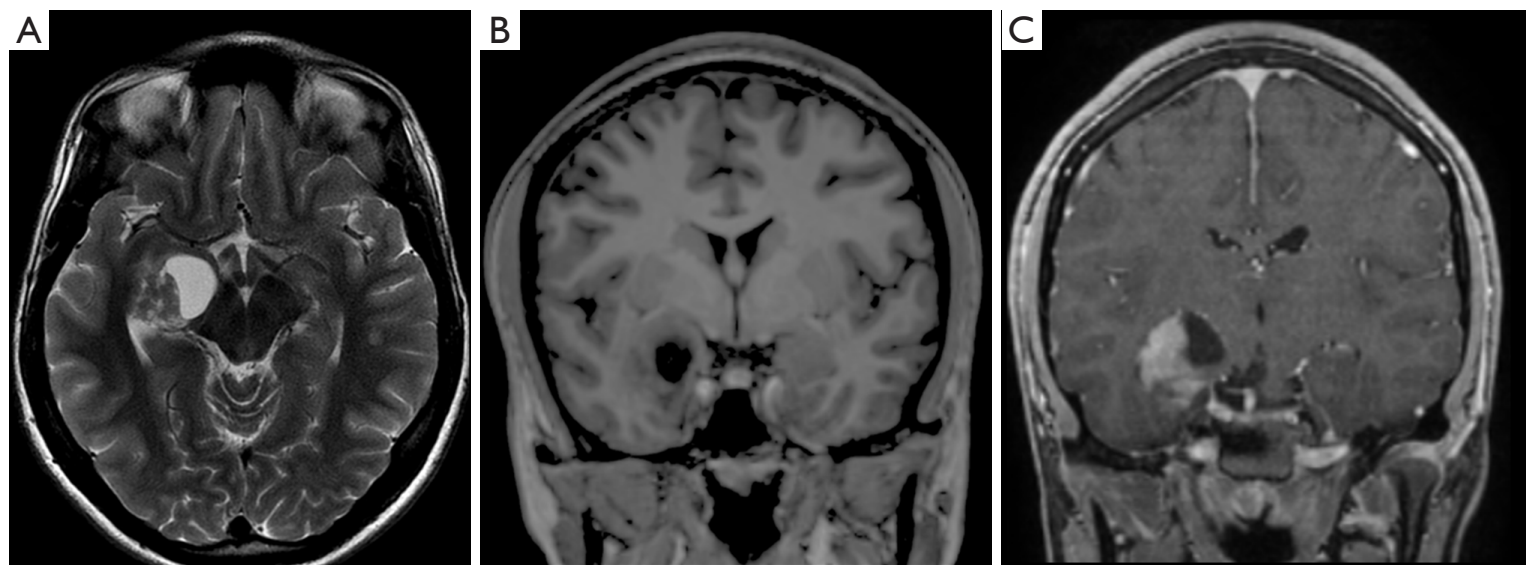

Figure 7 Male, 13 years old, diagnosis of ganglioglioma. Brain MRI: T2WI, T1WI, and T1W post-contrast (A,B,C) show a well-defined solid-cystic mass located in the right mesial temporal lobe with a solid mural nodule isointense to the cortex on T2WI, that enhances on postcontrast T1WI sequence (C). T1WI, T1-weighted image; T2WI, T2-weighted image.

recently described tumor of the CNS with an overall poor prognosis. They currently lack a WHO grade classification (3) and are most commonly observed in individuals below the age of 18. Patients' average survival is reported to be low, at only 22 months. The symptoms are those related to hydrocephalus due to accumulation of tumor within the subarachnoid space, although seizures, cranial nerve impairment, and ataxia may also be seen.

\section{Molecular features}

KIAA1549:BRAF fusion, $1 \mathrm{p}$ deletion or $1 \mathrm{p} / 19 \mathrm{q}$ codeletion and a mitogen-activated protein kinase (MAP-Kinase) pathway gene alteration without $I D H$ mutation are the characteristic molecular features of this tumor (73).

\section{Imaging features}

On MRI, this tumor often presents as diffuse intracranial and intraspinal lesions with nodular/thick leptomeningeal appearance and avid enhancement extending over the basal cisterns and brain and spinal surfaces. Presence of microcysts/nonenhancing small cyst-like lesions and dilation of perivascular spaces are commonly observed (3,74-76). Less frequently, non typical features, such as isolated spinal cord or brain lesions without clear leptomeningeal involvement, can be found (76). The main differential diagnosis includes inflammatoryinfection entities, particularly those that often show signs of meningitis with diffuse leptomeningeal enhancement, cisternal involvement, and hydrocephalus such as tuberculosis (77).

\section{Desmoplastic infantile tumors}

Desmoplastic infantile tumors (astrocytoma and gangliogliomas) (DIT) are uncommon intracranial tumors occurring mainly in patients younger than 2 years. These are considered $W H O$ grade $I$ tumors that tend to have a good prognosis, despite their aggressive appearance. They usually present with a rapid increase in the head circumference diameter (days to months) (78).

\section{Molecular features}

Desmoplastic infantile tumors usually harbor BRAF V600D and BRAF V600E mutations. Some uncommon aggressive forms with malignant transformation may present other molecular features such as TP53 mutation and ATRX deletions (78).

\section{Imaging features}

On imaging there is a large brain hemispheric mass with cystic and solid components. The solid component tends to be located more superficially in the tumor extending into the cortex and meningothelial tissues, commonly observed at the frontal and parietal lobes. Thickening and enhancement of the tumor adjacent meningitis associated with and calcifications may be present (79). The imaging appearance may be similar to glioblastoma, a $W H O$ grade $I V$ tumor that is usually associated with a worse prognosis. Diffusion-weighted imaging may be useful in distinguishing between infantile glioblastoma and DIT tumors, since infantile glioblastoma demonstrates restricted diffusion $(80,81)$. 

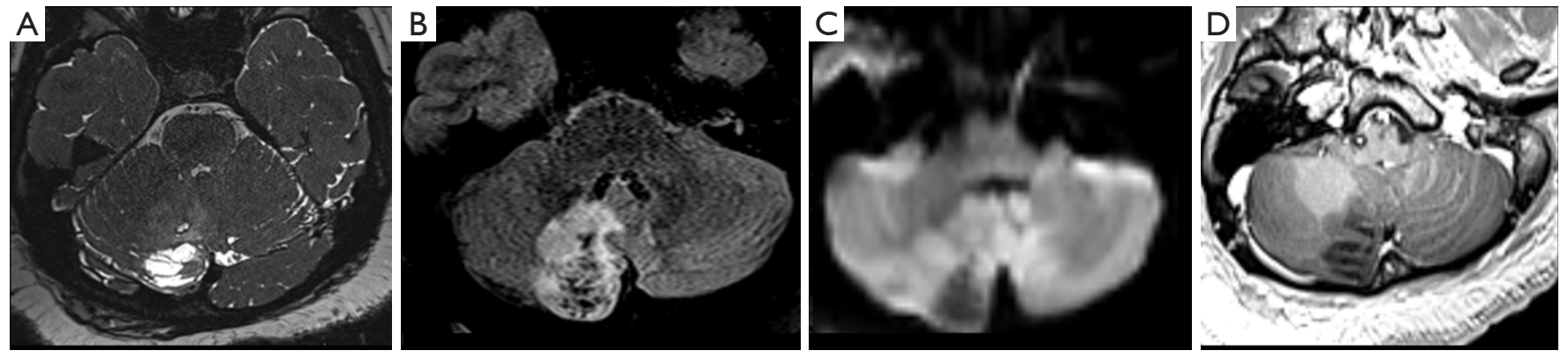

Figure 8 Male, 17 years old, diagnosis of dysplastic cerebellar gangliocytoma. Brain MRI: T2-FIESTA, FLAIR, DWI, and postcontrast T1WI (A,B,C,D) show a cortical lesion in the right cerebellar hemisphere, with a striated appearance. The lesion is hyperintense on T2WI (A) with areas of signal decrease on FLAIR (B), no areas of restriction on DWI (C), and no areas of enhancement on postcontrast T1WI (D). DWI, diffusion weighted imaging; FLAIR, fluid-attenuated inversion recovery; SWI, susceptibility weighted imaging; T1WI, T1weighted image; T2WI, T2-weighted image.

\section{DNET}

DNETs are slow-growing benign WHO Grade I glioneuronal tumors, typically diagnosed in children and young adults. They usually grow in deep or cortical gray matter extending to the juxtacortical white matter. Patients typically manifest longstanding, treatment-resistant partial seizures and neurological focal deficits. DNETs are commonly observed in temporal lobes, but can be found anywhere in the encephalon where gray matter is encountered. DNETs are often associated with focal cortical dysplasia, which may be observed as clearly separated from DNETs (Blumcke classification IIIb), or be contiguous to or mixed with the tumor $(82,83)$.

\section{Molecular features \\ Differentiating DNETs from astrocytomas and oligodendrogliomas can be challenging. Genetics could be a helpful tool, since DNETs are negative for $I D H$ mutations, TP53 mutations, and do not demonstrate $1 \mathrm{p} 19 \mathrm{q}$ co-deletion, while low-grade astrocytomas are usually $I D H$ mutated and oligodendrogliomas are often $I D H$-mutated and $1 \mathrm{p} 19 \mathrm{q}$ co-deleted (84).}

\section{Imaging features}

On imaging, DNETs typically present as a cortical or deep gray matter lesion with wedge shape and almost no surrounding edema. When they occur near bone structures, there may be scalloping or remodeling; erosion is not commonly observed. On T2WI, DNETs show hypersignal and a "bubbly" appearance, and on FLAIR a bright rim sign can be observed. Calcifications and gadolinium enhancement may or may not be present, and reduced diffusion is not commonly observed (Figure 9) $(85,86)$. The differential diagnoses include a tumefactive appearance of the perivascular space, but these are more often fully attenuating on FLAIR; multinodular and vacuolating neuronal tumors (MVNT) (87), more frequent in adults and not associated with seizures, low-grade gliomas, and other neuroglial tumors that may present with a "bubbly" appearance.

\section{Rosette-forming glioneuronal tumor (RGNTs)}

RGNT is an uncommon, usually midline neoplasm involving the mesencephalic aqueduct and/or the fourth ventricle. It is a WHO grade I tumor, composed of neuronalglial tissue characterized by biphasic architecture of glial and neurocytic components (88).

\section{Molecular features}

FGFR1 mutations may represent a molecular feature for this tumor entity. Unlike pilocytic astrocytomas, RGNTs do not show BRAF mutations (89).

\section{Imaging features}

On MRI these tumors are relatively well-circumscribed, but they might invade adjacent tissues, including the cerebellum, pons, and the pineal gland. They may present as solid tumors, with variable cystic components; the solid component may present a vivid and heterogeneous gadolinium enhancement (90). The main differential diagnosis includes inflammatory-infection entities, particularly those that present with a multicystic 

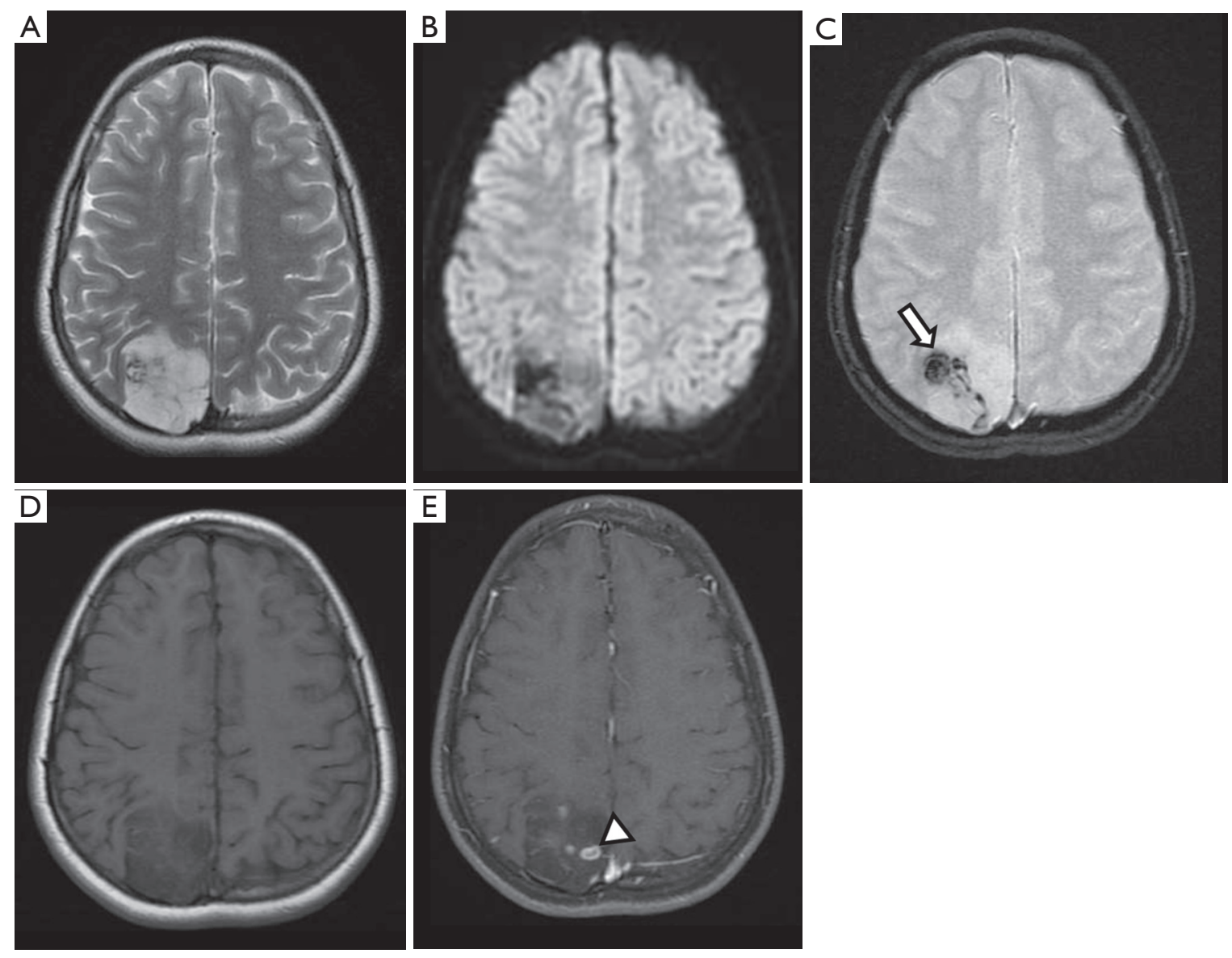

Figure 9 Female, 8 years old, diagnosis of DNET. Brain MRI: T2WI, DWI, SWI, T1WI, and T1W postcontrast (A,B,C,D,E) show a predominantly cortical lesion in the right occipital lobe hyperintense on T2WI, with a 'bubbly appearance,' and remodeling the adjacent inner table of the skull vault (A). There were no areas of restriction on DWI (B). Low-signal foci were observed on SWI, confirmed as components of calcium on a CT scan not shown (arrow, C). Some small areas of enhancement were noted (arrowhead, E). DNET, dysembryoplastic neuroepithelial tumor; DWI, diffusion weighted imaging; SWI, susceptibility weighted imaging; T1WI, T1-weighted image; T2WI, T2-weighted image.

appearance, such as tuberculosis, and also other low grade tumors including DNET, and pilocytic astrocytomas.

\section{Embryonal tumors-overview}

Embryonal tumors are aggressive, malignant, undifferentiated or poorly differentiated neoplasms of neuroepithelial origin that demonstrate an increased tendency to recur and propagate throughout the CNS via cerebrospinal fluid pathways.

The 2016 CNS WHO classification still categorizes these tumors as grade $I V$. However, several changes were made regarding how embryonal tumors should be subgrouped. First, and most importantly, medulloblastomas start to be mostly defined by their molecular profile, instead of the previous classification based on only their histological subtypes. Second, the subcategory primitive neuroectodermal tumors (PNETs) has been removed from the current lexicon. Third, the new entity embryonal tumor with multilayered rosettes (ETMR) was added to the 2016 WHO classification, grouping previous different embryonal tumor subtypes.

The rationale for the redistribution and regrouping of all these tumors was mainly driven by the perception that, in general, these tumors may share a similar molecular profile, which more often superimposes the histological features regarding demographics, aggressive behavior, potential treatments, and outcome $(3,91)$ (Table 4). 


\section{Medulloblastomas}

Medulloblastoma is the most common malignant solid tumor in children, representing the vast majority of all the embryonal tumors, more often in children older than 2 years and located in the posterior fossa.

\section{Molecular features}

New advancements in molecular profiling of these tumors led to establishing a classification that holds not only histologic features but also an integrated diagnosis based on four main molecularly defined subtypes: medulloblastoma Wingless (WNT)-activated, medulloblastoma Sonic hedgehog (SHH)-activated, medulloblastoma Group 3, and medulloblastoma Group 4. This reclassification constitutes the basis of new risk stratification schemes applied to current therapeutic clinical trials (3). Other molecular features not yet part of the classification, but with clinical importance and presenting clearly demographic distinctions, are the presence of MYCN, GLI2, and YAP1 amplification in children, and PTEN loss in neonates with $\mathrm{SHH}$ medulloblastomas. These changes are associated with increased rates of metastasis and poor outcome (92).

\section{Imaging features}

The most common MRI feature of medulloblastomas, regardless of the subtype, is the presence of restriction on DWI sequence (93), which is in accordance with the imaging appearance of all embryonal tumors (Figure 10). Other imaging findings that may help the distinction among the molecular subgroups mainly rely on the location and presence of enhancement of the tumors; these could offer an integrated interpretation of molecular results of medulloblastoma into clinical practice (94). Medulloblastomas in Groups 3 and 4 are predominantly distributed along the midline fourth ventricle, not infrequently with CNS dissemination at the moment of the diagnosis, while medulloblastoma $W N T$ are potentially localized in the cerebellar peduncle or in the cerebellopontine angle cistern and have low rates of CNS metastasis. More recent studies of $W N T$ medulloblastomas, however, have shown that these tumors are not as lateralizing as previously reported in studies with smaller cohorts $(93,95)$. Medulloblastomas $\mathrm{SHH}$ tend to arise from the cortex of the cerebellar hemispheres, presenting with a nodular or pyriform shape (Figure 11). The minimal or lack of enhancement of the solid part of the tumor is associated with the diagnosis of medulloblastomas in Group 4 (Figure 12). Spectroscopy shows an aggressive metabolite pattern, with elevated choline, lipid, and lactate peaks and the presence of taurine peaks may help to distinguish Group 3 and Group 4 subgroups, that present with higher peaks, from $\mathrm{SHH}$-activated tumors, which may show little or no taurine peak (96). The main differential diagnosis includes other embryonal tumors, particularly the Atypical Teratoid/Rhabdoid Tumor (AT/ RT), which tends to present in a younger age and with a more heterogeneous and aggressive imaging behavior and anaplastic ependymomas.

\section{$A T / R T$}

AT/RT is an uncommon $W H O$ grade $I V$ tumor, so named due to its histologic appearance, which contains an unusual mixture of primitive neuroepithelial, surface epithelial, and mesenchymal elements. Because of its primitive neuroepithelial elements, AT/RT might be misdiagnosed as medulloblastomas, but AT/RTs are often a diagnosis of infancy, not childhood (median age: 17 months). They are found outside the cerebellum in at least one third of cases, and are less likely to respond to treatment (97).

\section{Molecular features}

Ultimately, AT/RT is a genetic/molecular diagnosis, defined in $98 \%$ of cases by the deletion in chromosome 22 and loss of SMARCB1/INI1 expression in cytogenetic analysis of the tissue which also differentiate them from medulloblastomas $(91,98)$.

\section{Imaging features}

On imaging, AT/RT presents as a heterogeneous tumor with components of restriction on DWI affecting the CNS of the pediatric population, especially for patients under 4 years of age. These tumors are usually located in the midline or lateral aspect of posterior fossa with an intracanalicular component extending to the porus acusticus. These tumors may also be encountered in the supratentorial compartment, in the spine, or with a multicentric distribution. Cranial nerve involvement is also a possible location for these tumors (Figure 13) (99). Leptomeningeal seeding may be observed in up to $30 \%$ of cases, even at initial presentation, so neuraxis imaging evaluation should be considered in AT/RT-suspected cases. Necrosis, cystic formations, and hemorrhage are features that could be noted in AT/RTs (Figure 14). Although the sixth and seventh cranial nerves are most commonly involved with AT/RT, AT/RTs may occur also intrinsically to the third cranial nerve and present as an isolated third cranial nerve palsy. Spectroscopy 
Table 4 Summary table of embryonal tumors

\begin{tabular}{|c|c|c|c|c|c|c|}
\hline *Neoplasm & Subtypes & $\begin{array}{l}\text { Distinct imaging } \\
\text { features }\end{array}$ & Locations & $\begin{array}{l}\text { Histology and } \\
\text { molecular }\end{array}$ & $\begin{array}{l}\text { Grade } \\
(\mathrm{WHO})\end{array}$ & Outcome \\
\hline \multirow[t]{4}{*}{ Medulloblastoma } & WNT & $\begin{array}{l}\text { Midline location with } \\
\text { possible extension/ } \\
\text { origin from the } \\
\text { pontocerebellar } \\
\text { angle. Variable } \\
\text { component of } \\
\text { enhancement }\end{array}$ & $\begin{array}{l}\text { Cerebellum } \\
\text { (IV ventricle/ } \\
\text { vermis). Possible } \\
\text { pontocerebellar } \\
\text { angle location }\end{array}$ & $\begin{array}{l}\text { Classic, rarely } \\
\text { LCA, WNT } \\
\text { signaling, } \\
\text { MYC+ }\end{array}$ & ${ }^{\star \star \star \star} \mathrm{IV}$ & Good prognosis \\
\hline & $\mathrm{SHH}$ & $\begin{array}{l}\text { Nodulariform/ } \\
\text { multinodular } \\
\text { morphology or } \\
\text { cerebellar folie } \\
\text { "enlargement" } \\
\text { associated with } \\
\text { postcontrast } \\
\text { enhancement }\end{array}$ & $\begin{array}{l}\text { Cerebellar } \\
\text { hemispheres/extra- } \\
\text { axial extension }\end{array}$ & $\begin{array}{l}\text { Desmoplastic/ } \\
{ }^{\star \star} \mathrm{MBEN}, \mathrm{SHH} \\
\text { signaling, } \\
\text { MYCN+ }\end{array}$ & ${ }^{* * \star} \mathrm{IV}$ & $\begin{array}{l}\text { Good prognosis } \\
\text { (infants), } \\
\text { Intermediate } \\
\text { (others) }\end{array}$ \\
\hline & Group 3 & $\begin{array}{l}\text { Avid contrast } \\
\text { enhancement. } \\
\text { Presence of CSF } \\
\text { leptomeningeal } \\
\text { spread at the onset }\end{array}$ & $\begin{array}{l}\text { Cerebellum } \\
\text { (Vermis/IV ventricle) }\end{array}$ & $\begin{array}{l}\text { Classic/LCA, } \\
\text { MYC+++ }\end{array}$ & IV & Poor prognosis \\
\hline & Group 4 & $\begin{array}{l}\text { Mild or absence of } \\
\text { contrast enhancing. } \\
\text { Presence of CSF } \\
\text { leptomeningeal } \\
\text { spread at the onset }\end{array}$ & $\begin{array}{l}\text { Cerebellum } \\
\text { (Vermis/IV ventricle) }\end{array}$ & $\begin{array}{l}\text { Classic/LCA, } \\
\text { Minimal MYC or } \\
\text { MYCN }\end{array}$ & IV & $\begin{array}{l}\text { Intermediate } \\
\text { prognosis }\end{array}$ \\
\hline
\end{tabular}

*, Embryonal tumors are more often aggressive tumors located in the posterior fossa. There tumors are commonly hyperdense on CT scans. MR images show areas of restricted diffusion on DWI, hyperintensity on T1WI, and iso/hyperintensity on T2WI. Areas of enhancement on postcontrast T1WI vary and may be absent. **, MBEN, medulloblastoma with extensive nodularity - more common in infants. LCA, Large-cell anaplastic; ${ }^{* \star *}$, Although Grade IV more often these subtypes have a good prognosis. AT/RT, atypical teratoid/ rhabdoid tumor.

shows an aggressive metabolite pattern, with elevated choline, lipid, and lactate peaks. The differential diagnosis includes other embryonal tumors, particularly more aggressive presentations of medulloblastoma and anaplastic ependymomas.

\section{Other embryonal tumors}

The 2016 WHO classification has removed the previously nominated CNS PNETs from their lexicon $(3,91)$. The primary reason for the substantial change in this group of 

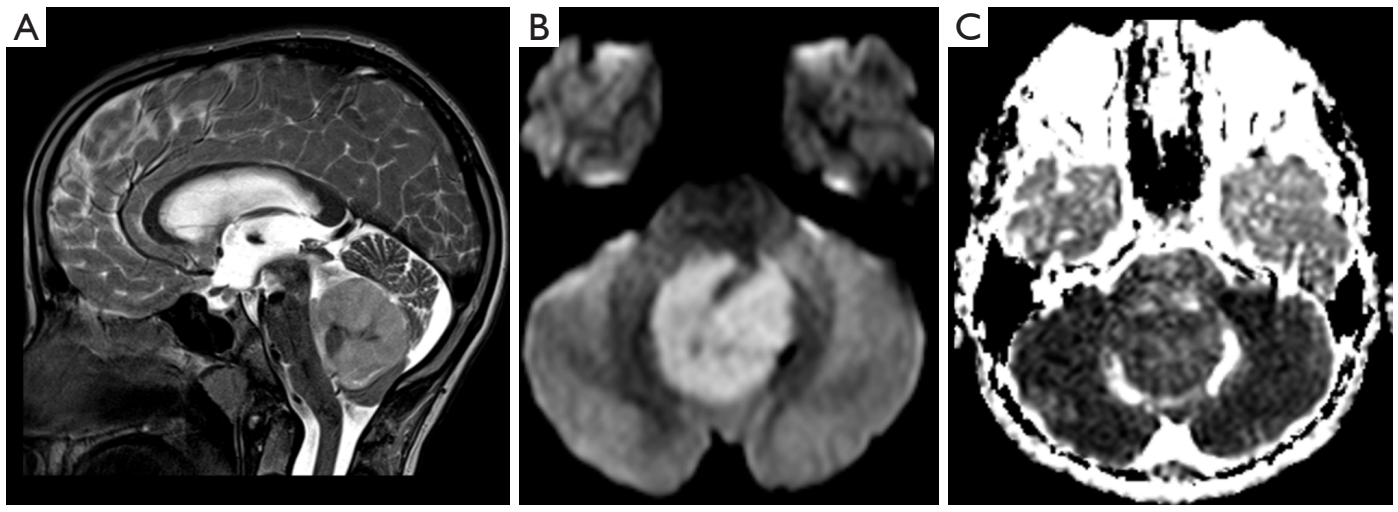

Figure 10 Female, 4 years old, diagnosis of medulloblastoma. Brain MRI: T2WI, DWI, and ADC map (A,B,C) show a well-delineated midline fourth ventricle mass, compressing the brainstem and the cerebellum, with restricted diffusion, confirmed in the ADC map (B,C), a hallmark of this tumor. ADC, apparent diffusion coefficient; DWI, diffusion weighted imaging; T1WI, T1-weighted image; T2WI, T2weighted image.
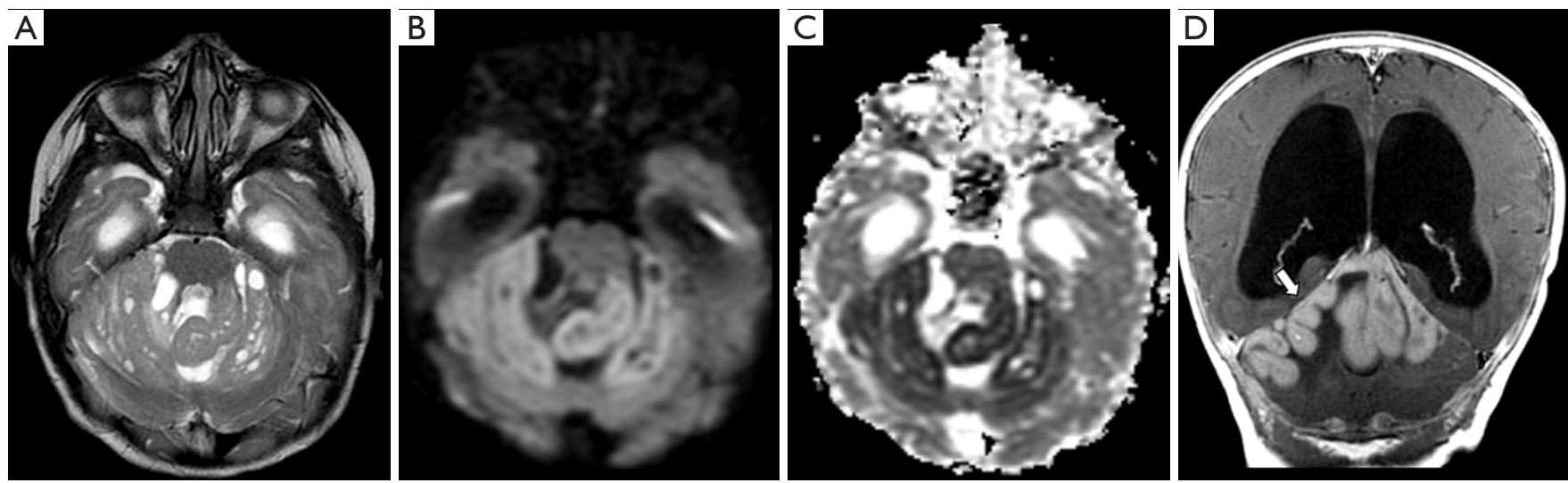

Figure 11 Male, 2 years old, diagnosis of medulloblastoma-SHH. Brain MRI: T2WI, DWI and ADC map, and T1WI post-contrast (A,B,C,D) show a bihemispheric and vermian cerebellar lesion, distorting and expanding the cerebellar cortex (A), presenting microcysts, and with restricted diffusion, confirmed in the $\mathrm{ADC}$ map $(\mathrm{B}, \mathrm{C})$, and with diffuse enhancement (D). ADC, apparent diffusion coefficient; DWI, diffusion weighted imaging; T1WI, T1-weighted image.

tumors was based on recent study results that discovered that among various subtypes, which had been previously described as different entities, several shared the same molecular profile: $C 19 M C$-altered. According to the recent classification three major groups should be considered: (I) the new genetically defined entity, embryonal tumor with multilayered rosettes (ETMR), C19MC-altered; (II) CNS embryonal tumor, NOS (not otherwise specified) for those tumors with absence of $C 19 M C$ amplification, thus defined based on "unique" histological results; and (III) medulloepithelioma, for the remaining part of the tumors that have histological features of medulloepithelioma, but do not express the $C 19 M C$-altered $(3,91)$.

\section{Molecular features}

The new entity, ETMR, C19MC-altered, is characterized by a high-level amplification of the chromosome 19 microRNA cluster $(C 19 M C)$ in the setting of a malignant pediatric brain tumor and is categorized as a $W H O$ grade $I V$ tumor. This new entity has replaced a "broad" group of tumors formerly known as ependymoblastoma or embryonal tumors with abundant neuropil and true rosettes (ETANTR), plus part of the medulloepithelioma tumors. 

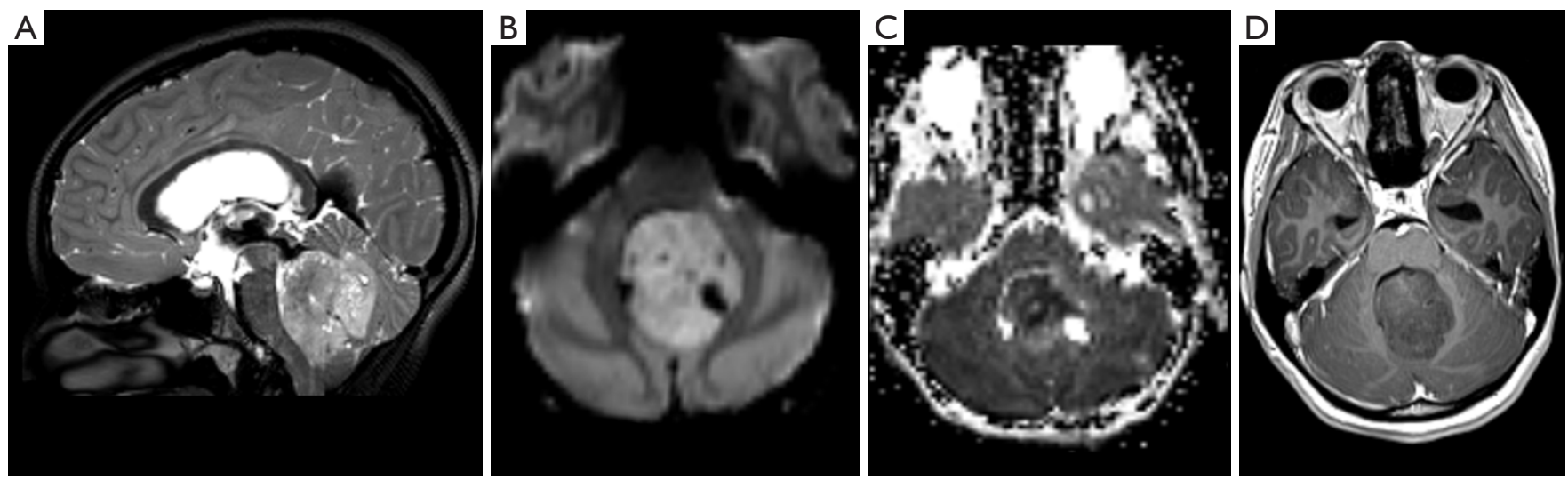

Figure 12 Male, 6 years old, diagnosis of medulloblastoma-Group 4. Brain MRI: T2WI, DWI and ADC map and T1WI post-contrast (A,B,C,D) show a well-delineated midline fourth ventricle and inferior vermian mass, compressing the brainstem anteriorly. The lesion has microcysts observed on T2WI (A) and restricted diffusion, confirmed in the ADC map (B,C). The lesion does not have significant enhancement, a characteristic feature of medulloblastoma-Group 4. ADC, apparent diffusion coefficient; DWI, diffusion weighted imaging; T1WI, T1-weighted image; T2WI, T2-weighted image.
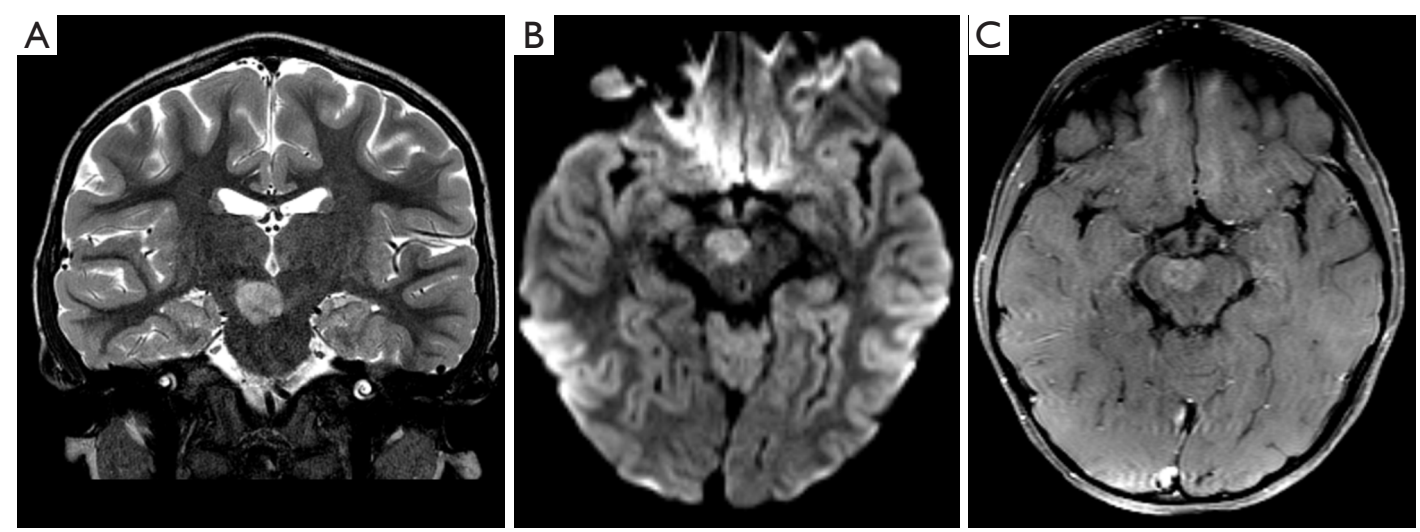

Figure 13 Male, 1 year old, diagnosis of AT/RT. Brain MRI: T2WI, DWI, and T1WI post-contrast (A,B,C) show a mass located in the right cerebral peduncle and interpeduncular cistern, hyperintense on T2WI (A), restricted on diffusion (B), and with enhancement on the post-contrast T1WI sequence (C). DWI, diffusion weighted imaging; T1WI, T1-weighted image; T2WI, T2-weighted image.

Most ETMRs occur in children younger than 4 years, are located in the cerebral hemispheres, and show an aggressive behavior (median survival: 0.8 years) (100).

\section{Imaging features}

On MRI, these are usually large mass-effect tumors, with diffuse signal heterogeneity, isointense or hyperintense signal on T2WI, and associated with contrast enhancement on postcontrast T1WI. They also present with components of internal necrosis and hemorrhage. Similar to other embryonal tumors, the DWI sequence showing restricted diffusion is a standard characteristic for this entity (101). ETMRs C19MC-altered tumors do not present a precise imaging hallmark for their diagnosis, and have a significant overlap appearance among themselves and with other embryonal tumors. Consequently, the hypothesis of ETMR C19MC-altered should be considered for any hypercellular and heterogeneous tumor of the CNS (supratentorial compartment, cerebellum, brainstem, and spinal cord) in the pediatric population, especially for patients under 4 years of age. 

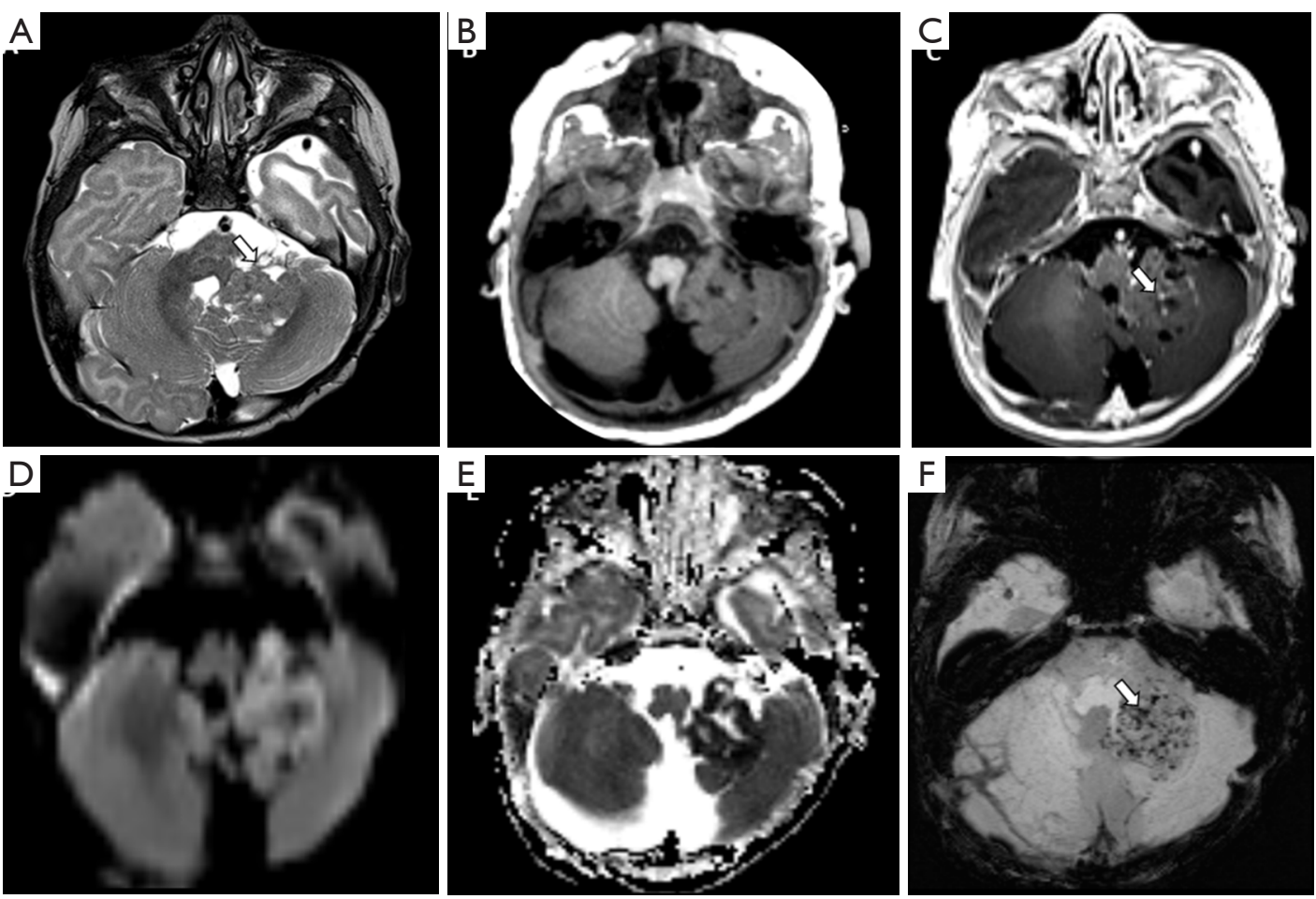

Figure 14 Female, 1 year old, diagnosis of AT/RT. Brain MRI: T2WI, T1WI, post-contrast T1WI, DWI, ADC, and SWI (A,B,C,D,E,F) show lobulated mass centered in the left Luschka foramen and middle cerebellar peduncle with a heterogeneous signal on T2WI and microcysts (A), minimal enhancement on the post-contrast T1WI (arrow, C) restricted on diffusion (D), confirmed on the ADC map (E) with some components of hemorrhage, characterized by small low-signal foci on SWI, and confirmed on a CT scan not shown (arrow, F). ADC, apparent diffusion coefficient; DWI, diffusion weighted imaging; T1WI, T1-weighted image; T2WI, T2-weighted image.

\section{Ependymal tumors}

Ependymoma is a neuroepithelial neoplasm accounting for $10 \%$ of pediatric brain tumors. These tumors typically occur in the posterior fossa, following the supratentorial compartment and, less frequently, the spine. In the past 2 decades, infratentorial, supratentorial, and spinal ependymomas have been shown to be biologically distinct entities that have different cells of origin. Spinal cord ependymomas (classic and myxopapillary) are very uncommon in children. Posterior fossa ependymomas can be split into two groups: Posterior Fossa A (PFA) and Posterior Fossa B (PFB).

\section{Molecular features}

The PFA ependymoma is commonly located in the lateral recess of very young children and is associated with a poor prognosis. This tumor's hallmark is promoter hypermethylation and disruption in $H 3 \mathrm{~K} 27$ trimethylation.
PFB ependymomas are commonly observed in the midline/ obex, are more often diagnosed in older children and adolescents, and tend to have a better prognosis. Presence of RELA or YAP1 (yes-associated protein 1) mutations are related to supratentorial ependymomas $(17,102)$.

Supratentorial ependymomas account for $30 \%$ of ependymomas and are commonly observed near ventricles, although they can be found anywhere in the brain hemispheres, with a frontoparietal predominance. RELAsubgroup ependymoma usually occurs in older children, accounts for $70 \%$ of supratentorial ependymomas, and is associated with a poor survival rate (Figure 15), while the YAP1 subgroup is typical in infants and is associated with a very good prognosis (103).

\section{Imaging features}

MRI usually shows posterior fossa ependymomas extending through the foramina of Luschka and Magendie, encasing 

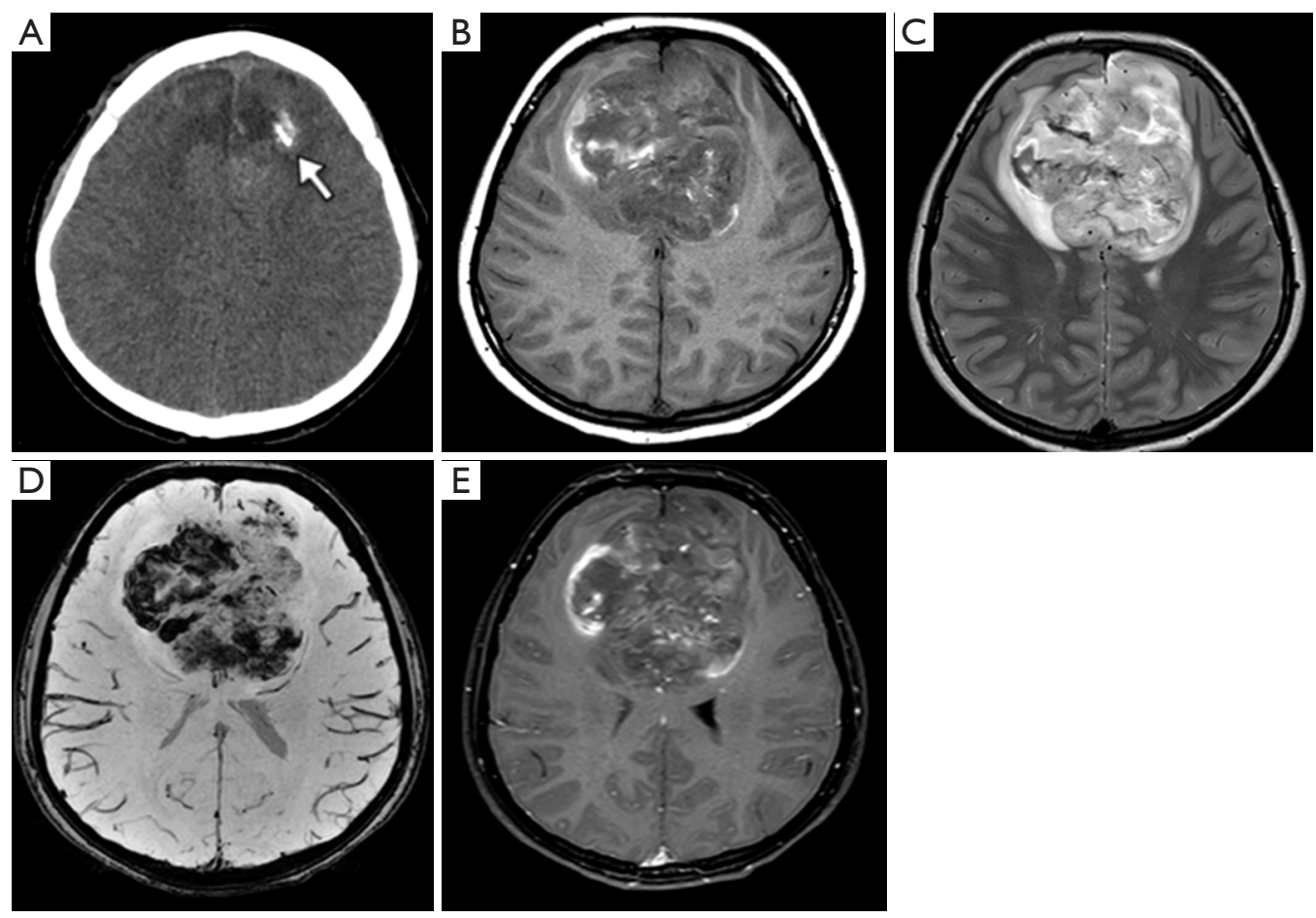

Figure 15 Male, 15 years old, diagnosis of supratentorial ependymoma RELA-fusion. Brain CT shows a median bi-frontal brain lesion with some focus of calcification on the left (A, arrow). Brain MRI (B,C,D,E). T1WI shows a lobulated tumor predominantly hypointense with some hyperintense peripheral areas (B). The T2WI shows a diffuse hyperintensity with some central components, with more hyperintensity related with cystic/necrotic areas (C), susceptibility-weighted imaging demonstrates multiple and confluent hypointense foci inside the tumor compatible with hemorrhage (D), and post-gadolinium contrast T1-weighted shows subtle, sparse foci of enhancement (E). T1WI, T1-weighted image.

neurovascular structures. This "plastic" behavior contributes to the late clinical manifestation of posterior fossa ependymomas compared with the clinical manifestations of other posterior fossa tumors.

Ependymomas show a heterogeneous high T2WI signal intensity and calcification observed in susceptibility sequences in $50 \%$ of cases; hemorrhage may be observed, although it is not a common finding. On DWI, they show an intermediate pattern of restriction when compared to pilocytic astrocytomas and medulloblastomas. Postcontrast T1WI shows avid enhancement of the solid component along with several nonenhancing cystic and/or necrotic components (Figure 16) (104). The differential diagnosis includes embryonal tumors; particularly those with a "plastic" appearance and pilocytic astrocytomas.

\section{Choroid plexus tumors}

Choroid plexus tumors are rare intraventricular tumors of neuroectodermal origin, accounting for $2-4 \%$ of brain tumors in children. Tumor resection is reported to be curative for choroid plexus papillomas (CPP), while choroid plexus carcinomas are associated with recurrence and dissemination. According to the 2016 WHO classification, they can be classified into 3 subtypes: CPP (WHO grade I), atypical choroid plexus papilloma (aCPP, WHO grade $I I$ ), and choroid plexus carcinoma (CPC, WHO grade III). Imaging alone does not allow distinction between these neoplasms.

Choroid plexus papillomas are a rare intraventricular tumor (WHO grade I), more often observed in the pediatric population $(85 \%$ observed in patients below the age of 5 years). The overall prognosis for patients with CPP is good, with a 5 -year survival rate of $97 \%$. The main site for CPP in children is the supratentorial region, although it might be found also in the fourth ventricle. Atypical CPPs show intermediate clinical and pathological features between the much more common $W H O$ grade I CPP and the rare 

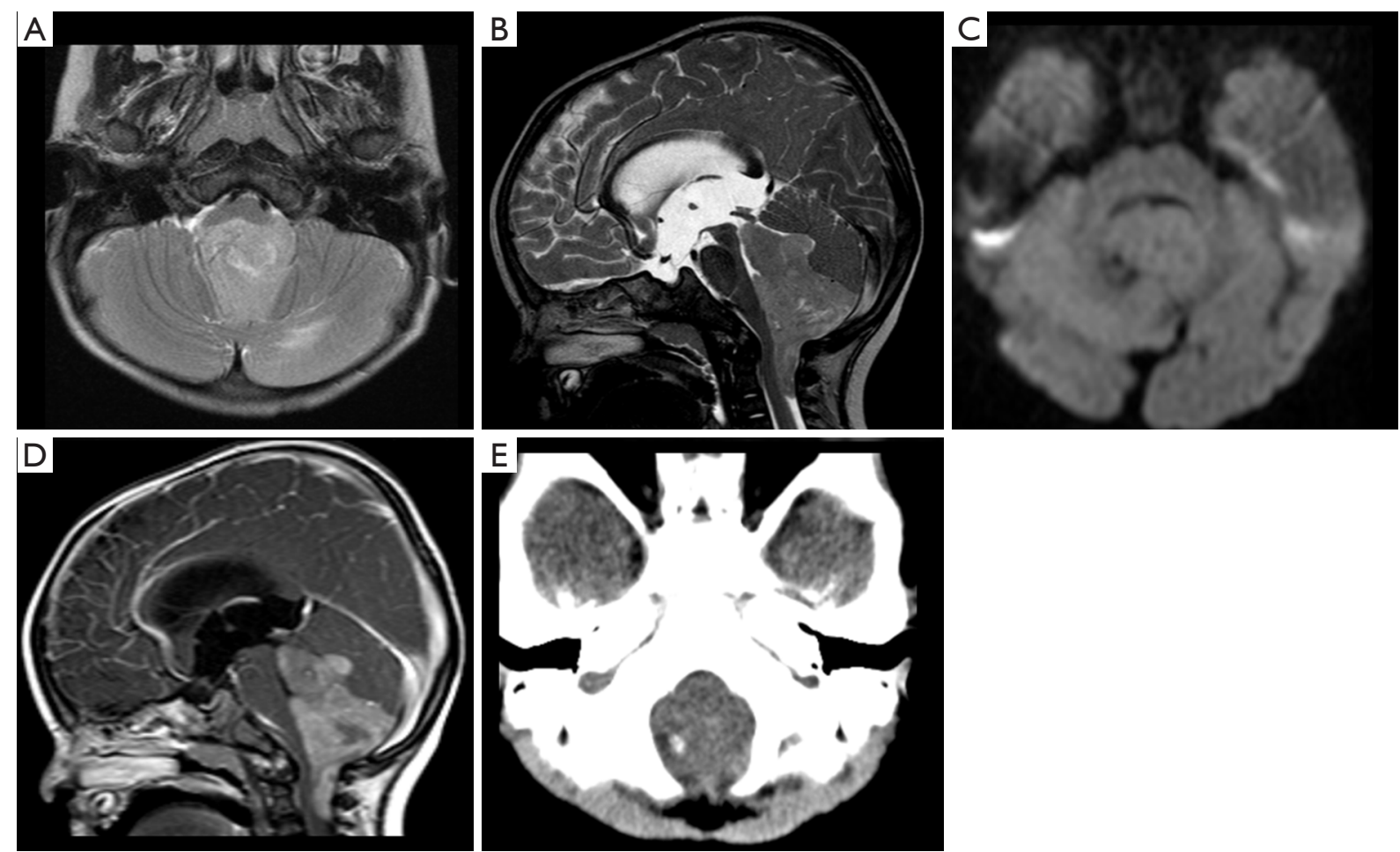

Figure 16 Female, 7 years old, diagnosis of PFB-ependymoma. Brain MRI: Axial T2WI, Sagittal T2WI, DWI, and post-contrast Sagittal T1WI. CT scan (E). A midline fourth ventricle mass, compressing the brainstem and the cerebellum arising from the Magendie and Luschka recesses, with a "plastic" appearance shows hyperintense signal on T2WI (A,B), no areas of restriction on DWI (C), enhancement on the post-contrast T1WI sequence (D), and a small focus of calcification on the CT scan (E). DWI, diffusion weighted imaging; T1WI, T1-weighted image; T2WI, T2-weighted image.

WHO grade II CPC. aCPP is characterized by two or more mitoses per 10 randomly selected high-power fields.

Choroid plexus carcinoma represents a malignant WHO grade III tumor with a median age of occurrence of 1 year, with more than $75 \%$ diagnosed before the age of 5 years. It accounts for up to $4 \%$ of pediatric brain tumors, is associated with Li-Fraumeni syndrome, and has a poor prognosis; it has a 5 -year survival rate of $26-43 \%$. CPC are mainly located within the ventricles, although extraventricular sites have been reported in the literature. Despite the data regarding the biologic and genetic makeup of pediatric CPC, there remain numerous barriers to understanding the best treatment strategies.

\section{Molecular features}

Although some molecular biomarkers enhance molecular differences of CPTs the histological grading is still the most reliable method to differentiate aggressive (CPC) from CPP and aCPP. The prediction of clinical outcome and potential group distinction among some CPTs could be improved by analysis of DNA methylation profile and the status of TP53 mutation (105).

\section{Imaging features}

All subtypes of choroid plexus tumors may demonstrate CSF dissemination; therefore, imaging of the entire neuraxis is always recommended $(106,107)$. Moreover, imaging features alone may not be enough for differentiating these subtypes of tumors and the differential should be always considered from the imaging point of view (108).

In general terms, CPPs are usually well-defined lobulated masses that may present a cystic component and a solid and vivid enhancement in the solid part (Figure 17); calcification is not a hallmark but can be found in some cases. The transformation from a CPP to a CPC has been reported in a small number of cases $(107,108)$. Differentiation between both neoplasms is commonly challenging from the imaging point of view; although CPCs tend to be larger, more 

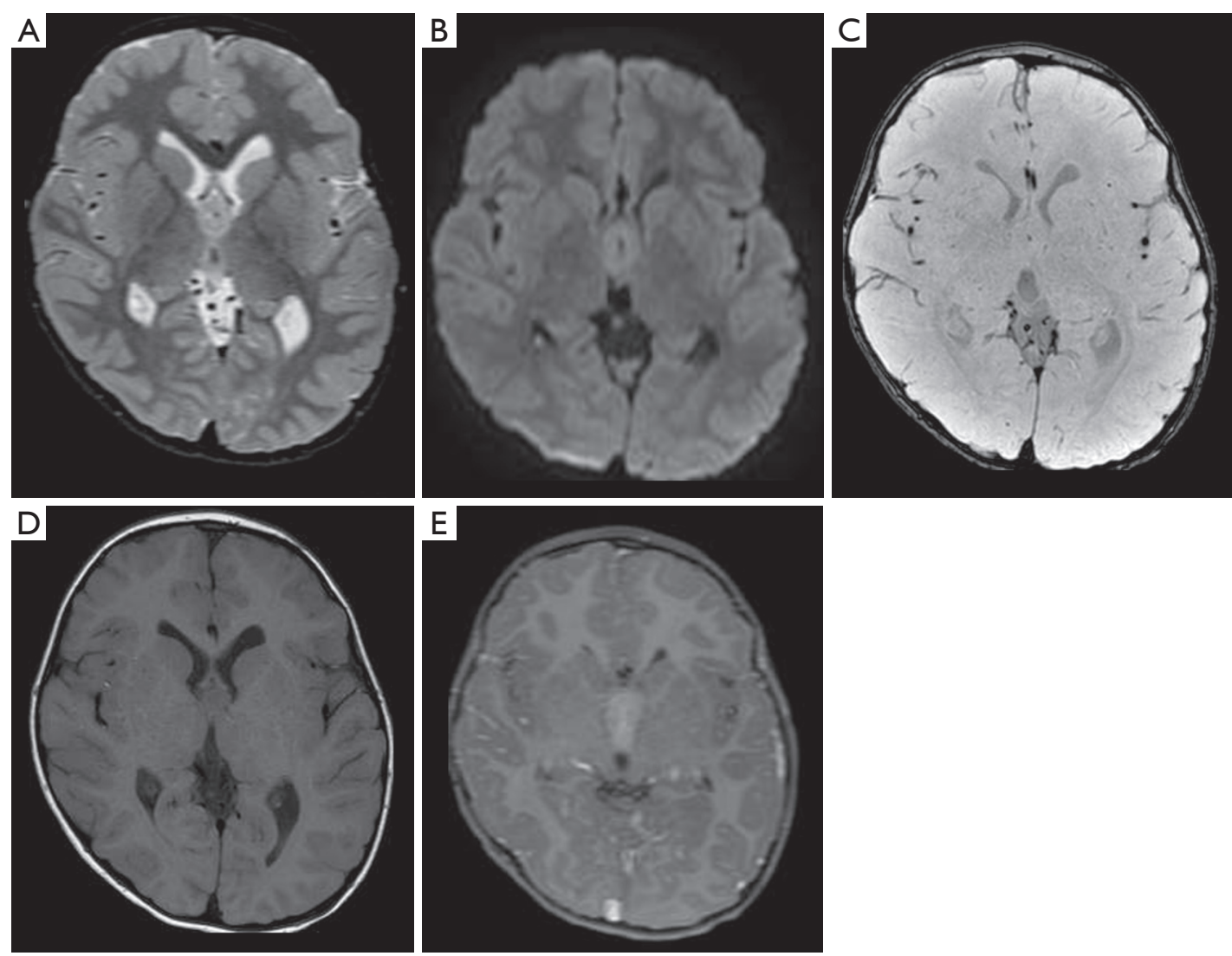

Figure 17 Female, 18 years old, diagnosis of choroid plexus papilloma. Brain MRI: Axial T2WI, DWI, SWI, T1WI, and postcontrast T1WI (A,B,C,D,E) show a mass located in the III ventricle, hyperintense on T2WI (A), without restricted diffusion (B), and with enhancement on the postcontrast T1WI (E). T1WI, T1-weighted image; T2WI, T2-weighted image.

infiltrative, and more heterogeneous associated with areas of necrosis. Moreover, even though both neoplasms may disseminate, CPCs are more often associated with CNS axis dissemination/metastasis (108).

\section{GCTs and differential diagnosis in the sellar region and pineal gland}

Intracranial GCTs are uncommon and biologically diversified CNS tumors. These neoplasms correspond to $2-3 \%$ of all pediatric brain tumors in Western countries, and have a higher frequency in East Asian countries, with incidence around $10 \%$. There is no explanation so far for this geographic and ethnic diversity between Asian and Western series (109). GCT onset is often observed during childhood, with the vast majority occurring before the age of 20 years (110-114). Moreover, a variability of the peak of incidence is also observed among the overall GCT, and can be separated by this tumor's histology features from non- germinomatous germ cell tumors (NGGCTs), which tend to occur in younger children, whereas pure germinomas occur more often in older children and adolescents (115).

CNS GCTs usually originate in the suprasellar and pineal regions. Less frequently, non-midline intracranial structures, including the basal ganglia and thalami may appear, and rarely the tumor may arise primarily in the spinal cord or the cerebellum (115-119). Males have an overall higher incidence of CNS GCTs than females. Differences among the sexes are also observed in the locations of the CNS tumor: $70 \%$ of males have their tumors appearing in the pineal gland, and $75 \%$ of females have their tumors located in the suprasellar region (120).

\section{Imaging features}

The GCT diagnosis should be always included in the differential of enhancement and thickened pituitary stalk and among pineal masses during childhood (121). 
GCTs are often well-delineated and lobulated lesions, demonstrating iso- or hyperdensity on CT, with solid and cystic components, and demonstrating signal heterogeneity in all MRI sequences, with areas of enhancement. Presence of calcification and hemorrhage foci are often observed on CT and/or MRI studies (122). Particularly for GCTs arising in the pineal region, an analysis of the morphology of the central physiological calcification of the pineal (after 4 years) may be helpful. Unlike neoplasms arising from the primary pineal cells (pineoblastoma and pineocytomas), the central physiologic calcification of the pineal tents to be preserved (unified, and centrally located) in GCTs (123). Other imaging details based on particular sequences include the restriction on DWI for the solid parts of the tumors, and the $1 \mathrm{H}$ MRS demonstrating abnormal peaks of lipids in $1.33 \mathrm{ppm}(124,125)$. Differences between pure and mixed GCTs are also described and include differences of sizes, morphology (micro versus macrolobulated), and the presence of components of hemorrhage, more commonly observed in mixed GCTs (Figure 18) (114).

\section{Post-treatment changes and secondary tumors}

Imaging analysis of post-treatment changes in the context of brain tumors is often a challenging task for radiologists in clinical practice. Several differences between subtypes of pediatric and adult brain tumors and a greater heterogeneity of the neoplasms in children make interpretation of these changes even more complicated. Although challenging, the ability to distinguish residual or recurrent tumors from post-treatment entities, such as radiation-induced lesions, may have a vital impact on the patient's outcome.

For this reason, an international panel of working groups with a focus on pediatric neuro-oncology was formed, called the Response Assessment in Pediatric Neuro-Oncology (RAPNO) (126), and imaging guidelines were created (127-129).

Brain changes can be related to several causes, including most frequently surgery-related lesions, radiation-induced lesions, and chemotherapy-induced lesions.

Regarding post-surgery imaging analysis, the MRI study must be obtained as soon as possible after surgery in order to take steps to reduce parenchymal changes.

The consensus recommendations include MRI studies performed within 72 hours postoperatively, thus bypassing the formation of scars. If there are extensive parenchymal postsurgical changes that may complicate the analysis of the residual tumor, a second brain MRI should be performed approximately 2-3 weeks after intervention (130).

Regarding radiation-related lesions, it is important to remember that these lesions may appear in both early and late stages of the treatment. Radiation-related lesions are caused by a variable compound of vascular and parenchymal damage resulting in tumoral necrosis or even necrosis in the normal parenchyma.

During the early stage, the most common imaging features are the presence of vasogenic edema (T2WI hyperintense) and new areas of contrast enhancement, both of which are frequently observed around the radiation area. In the late stage, one important process that may appear is radiation necrosis. Radiation necrosis represents a serious condition for the patient and results from a pathologic process of necrosis formation into the brain. There are two main factors associated with radiation necrosis: (I) time elapsed since radiation exposure (around 2 years of radiation), and (II) the radiation dose (exponential increasing after 62 Gy of dose) (131). Imaging features may vary, but the most remarkable is the presence of a distinct enhancement area along the margin of the tumor resection with an internal appearance of the so-called "soap-bubble." Advanced MRI techniques are crucial for distinguishing tumors from radiation necrosis. Unlike neoplasms, radiation necrosis often shows decreased $\mathrm{rCBV}$ on perfusion and elevated lipid/lactate peaks on $1 \mathrm{H}$ MRS. The areas of enhancement commonly disappear throughout followup imaging studies (131). DWI can be also very useful for the surveillance of relapse tumors, particularly embryonal tumors, since it can depict CNS relapses with higher accuracy than post-contrast $\mathrm{T} 1$ sequences, even after several years of surveillance (132).

Other lesions observed in the late stage, after radiation, are vascular proliferative lesions. On imaging these lesions are characterized by the presence of capillary telangiectasia and cavernous malformations (cavernomas), and they may present with an aggressive appearance, mimicking tumors (133).

\section{Future perspectives (cIMPACT-NOW)}

cIMPACT-NOW (the Consortium to Inform Molecular and Practical Approaches to CNS Tumor Taxonomy) is a consortium created to make recommendations on potential advances in the field of CNS tumor classification. In the last meeting in Utrecht in September 2019, the topics covered were the definitions of "new" entities and revised definitions of "old" entities (134).

The cIMPACT-Utrecht endorsed the clinical utility 

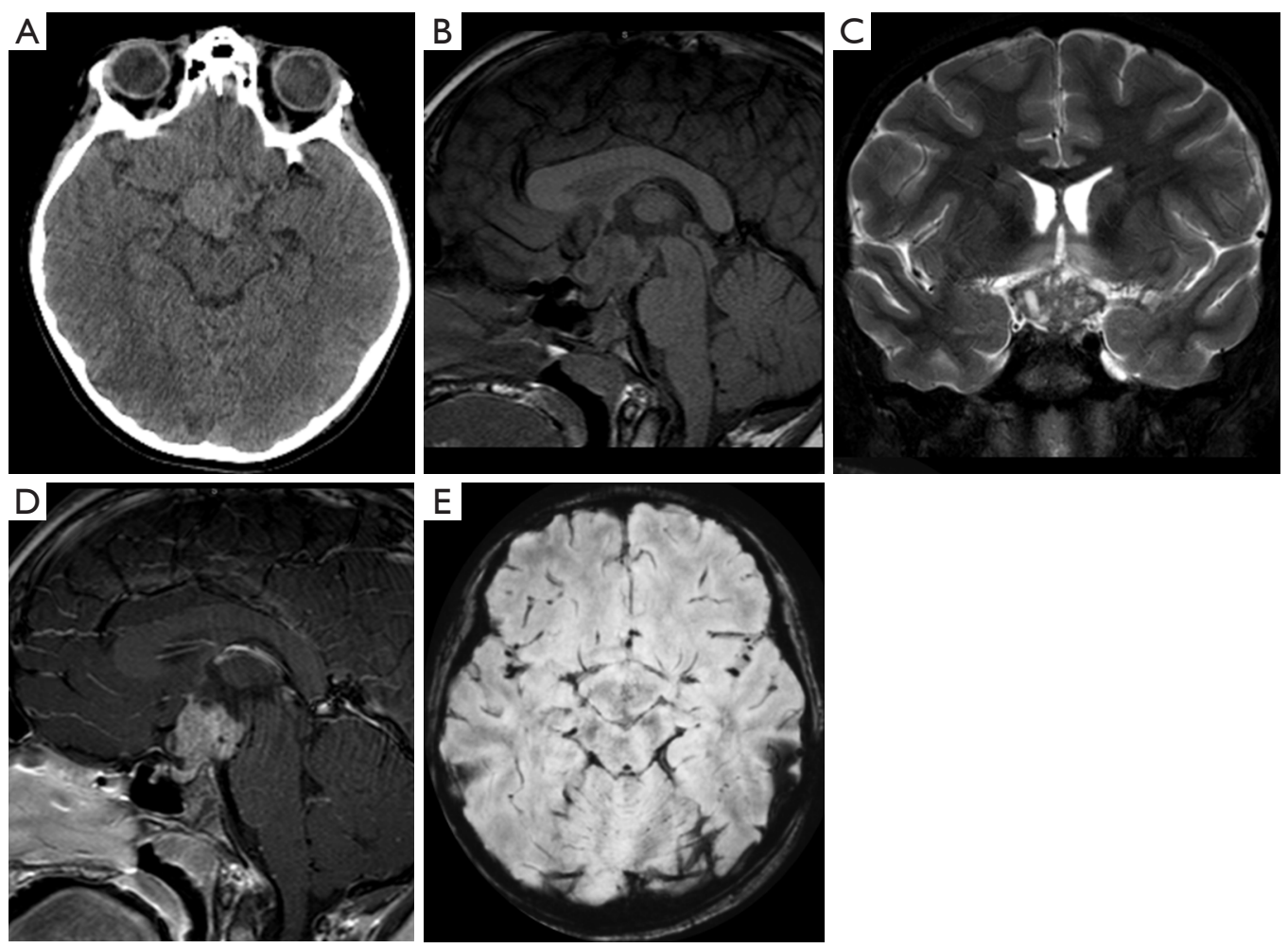

Figure 18 Male, 9 years old, diagnosis of pure germ cell tumor (germinoma). CT scan (A). Brain MRI (B,C,D,E). Sagittal T1WI, coronal T2WI, sagittal post-contrast T1WI, and axial SWI sequences. A suprasellar mass enlarging the pituitary stalk was noted. The mass has a lobulated morphology, mild hyperdense (A), hypointense on T1WI (B), hyperintense with microcystis on T2WI (C), homogeneous enhancement (D), and no areas of calcification or hemorrhage (E). T1WI, T1-weighted image; T2WI, T2-weighted image.

of two-list approaches for a range of pediatric glial/ glioneuronal tumors. This panel would apply a histologically and genetically defined list of tumors, which can be combined into an integrated diagnosis. For instance, the ependymomas would be categorized by anatomic site and molecular characterization (supratentorial ependymoma, RELA fusion-positive).

Tumor nomenclature is also under evaluation for improvement and standardization. It was suggested that names should be as simple as possible, and only location, age, or genetic modifiers with clear clinical utility should be adopted. As an example, chordoid gliomas which occur predominantly in the third ventricle, the localization information should be deleted from its nomenclature since it provides a mechanism to characterize the entity and is therefore not necessary in the name itself (134).

The cIMPACT-Utrecht committee agreed that the methylome profile may offer reliability to identify many CNS tumor types and subtypes since it has shown to provide powerful information for the classification and diagnosis of these tumors. Another modification supported by the last cIMPACT meeting was that all WHO CNS tumor grades should switch to Arabic numerals to decrease the possibility of typographical error that could address clinical consequences.

There are some particular modifications that may be implemented for some tumors most frequently observed in the pediatric population.

* Diffuse glioma, H3.3 G34-mutant: It should be listed in future classifications with the diffuse glial tumors as a novel tumor type, distinct from the established types of IDH-mutant and IDH-wildtype gliomas, as well as from the $H_{3} \mathrm{~K} 27 \mathrm{M}$-mutant diffuse midline gliomas.

* Polymorphous Low-grade Neuroepithelial Tumor of The Young (PLNTY): future classifications should consider placing either within the group of "Neuronal and mixed neuronal-glial tumors", or more specifically, in the family of "Pediatrictype glial tumors and glioneuronal tumors." These 
tumors may present some characteristic imaging findings including well-circumscribed lesions with presence of macroscopic calcification and a cystic component located peripherally/subcortically mainly in the posteroinferior temporal lobe. The pattern of prominent central calcification seen in these tumors is distinctly unusual $(135,136)$.

* PFA ependymoma: The presence of chromosome 1q gain is recognized as an adverse prognostic factor among pediatric-type/PFA tumors. The subsequent $\mathrm{WHO}$ terminology for ependymomas may differ from what has been proposed so far.

\section{Conclusions}

Primary pediatric brain tumors have variable clinical and imaging presentations, which may pose a challenge for the radiologist's diagnosis. The ability to identify the most common imaging phenotypes underlying molecular properties, as well as the features of aggressiveness, paves the way for early diagnosis for these children. Understanding the unique imaging features of types of tumors also supports appropriate therapies based on distinct characteristics of each subtype of tumor.

\section{Acknowledgments}

We thank Lydia Sheldon for helpful editing suggestions. Funding: None.

\section{Footnote}

Provenance and Peer Review: This article was commissioned by the Guest Editor (Felice D'Arco) for the series "Pediatric Neuroradiology for Trainees and Fellows: An Updated Practical Guide" published in Translational Pediatrics. The article has undergone external peer review.

Reporting Checklist: The authors have completed the Narrative Review reporting checklist. Available at http:// dx.doi.org/10.21037/tp-20-285

Peer Review File: Available at http://dx.doi.org/10.21037/tp20-285

Conflicts of Interest: Both authors have completed the ICMJE uniform disclosure form (available at http:// dx.doi.org/10.21037/tp-20-285). The series "Pediatric
Neuroradiology for Trainees and Fellows: An Updated Practical Guide" was commissioned by the editorial office without any funding or sponsorship. The authors have no other conflicts of interest to declare.

Ethical Statement: The authors are accountable for all aspects of the work in ensuring that questions related to the accuracy or integrity of any part of the work are appropriately investigated and resolved.

Open Access Statement: This is an Open Access article distributed in accordance with the Creative Commons Attribution-NonCommercial-NoDerivs 4.0 International License (CC BY-NC-ND 4.0), which permits the noncommercial replication and distribution of the article with the strict proviso that no changes or edits are made and the original work is properly cited (including links to both the formal publication through the relevant DOI and the license). See: https://creativecommons.org/licenses/by-nc-nd/4.0/.

\section{References}

1. Ostrom QT, Cioffi G, Gittleman H, et al. CBTRUS Statistical Report: Primary Brain and Other Central Nervous System Tumors Diagnosed in the United States in 2012-2016. Neuro Oncol 2019;21:v1-100.

2. de Blank PM, Ostrom QT, Rouse C, et al. Years of life lived with disease and years of potential life lost in children who die of cancer in the United States, 2009. Cancer Med 2015;4:608-19.

3. Louis DN, Perry A, Reifenberger G, et al. The 2016 World Health Organization Classification of Tumors of the Central Nervous System: a summary. Acta Neuropathol 2016;131:803-20.

4. Korshunov A, Ryzhova M, Hovestadt V, et al. Integrated analysis of pediatric glioblastoma reveals a subset of biologically favorable tumors with associated molecular prognostic markers. Acta Neuropathol 2015;129:669-78.

5. Zhang J, Wu G, Miller CP, et al. Whole-genome sequencing identifies genetic alterations in pediatric lowgrade gliomas. Nat Genet 2013;45:602-12.

6. Khuong-Quang D-A, Buczkowicz P, Rakopoulos P, et al. K27M mutation in histone $\mathrm{H} 3.3$ defines clinically and biologically distinct subgroups of pediatric diffuse intrinsic pontine gliomas. Acta Neuropathol 2012;124:439-47.

7. Sturm D, Pfister SM, Jones DTW. Pediatric Gliomas: Current Concepts on Diagnosis, Biology, and Clinical Management. J Clin Oncol 2017;35:2370-7. 
8. Gonçalves FG, Alves CAPF, Vossough A. Updates in Pediatric Malignant Gliomas. Top Magn Reson Imaging 2020;29:83-94.

9. Buczkowicz P, Bartels U, Bouffet E, et al. Histopathological spectrum of paediatric diffuse intrinsic pontine glioma: diagnostic and therapeutic implications. Acta Neuropathol 2014;128:573-81.

10. Packer RJ, Pfister S, Bouffet E, et al. Pediatric lowgrade gliomas: implications of the biologic era. Neuro Oncol.2017;19:750-61.

11. Chalil A, Ramaswamy V. Low Grade Gliomas in Children. J Child Neurol 2016;31:517-22.

12. Ryall S, Tabori U, Hawkins C. Pediatric low-grade glioma in the era of molecular diagnostics. Acta Neuropathol Commun 2020;8:30.

13. Ostrom QT, Gittleman H, Liao P, et al. CBTRUS Statistical Report: Primary brain and other central nervous system tumors diagnosed in the United States in 20102014. Neuro Oncol 2017;19:v1-88.

14. Loh JK, Lieu AS, Chai CY, et al. Arrested growth and spontaneous tumor regression of partially resected lowgrade cerebellar astrocytomas in children. Childs Nerv Syst 2013;29:2051-5.

15. Paldino MJ, Faerber EN, Poussaint TY. Imaging tumors of the pediatric central nervous system. Radiol Clin North Am 2011;49:589-616, v.

16. Bornhorst M, Frappaz D, Packer RJ. Pilocytic astrocytomas. Handb Clin Neurol 2016;134:329-44.

17. AlRayahi J, Zapotocky M, Ramaswamy V, et al. Pediatric Brain Tumor Genetics: What Radiologists Need to Know. Radiographics 2018;38:2102-22.

18. D'Arco F, Khan F, Mankad K, et al. Differential diagnosis of posterior fossa tumours in children: new insights. Pediatr Radiol 2018;48:1955-63.

19. Koeller KK, Rushing EJ. From the archives of the AFIP: pilocytic astrocytoma: radiologic-pathologic correlation. Radiographics 2004;24:1693-708.

20. Chourmouzi D, Papadopoulou E, Konstantinidis M, et al. Manifestations of pilocytic astrocytoma: a pictorial review. Insights Imaging 2014;5:387-402.

21. Taylor T, Jaspan T, Milano G, et al. Radiological classification of optic pathway gliomas: experience of a modified functional classification system. Br J Radiol 2008;81:761-6.

22. Ding C, Tihan T. Recent Progress in the Pathology and Genetics of Pilocytic and Pilomyxoid Astrocytomas. Balkan Med J 2019;36:3-11.

23. Komotar RJ, Burger PC, Carson BS, et al. Pilocytic and pilomyxoid hypothalamic/chiasmatic astrocytomas. Neurosurgery 2004;54:72-9; discussion 79-80.

24. Alkonyi B, Nowak J, Gnekow AK, et al. Differential imaging characteristics and dissemination potential of pilomyxoid astrocytomas versus pilocytic astrocytomas. Neuroradiology 2015;57:625-38.

25. Horbinski C. To BRAF or not to BRAF: is that even a question anymore? J Neuropathol Exp Neurol 2013;72:2-7.

26. Forbes K. Osborn, Salzman, Barkovich et al.: Diagnostic imaging: brain. 2nd edition. Neuroradiology 2012;54:269.

27. Lind-Landström T, Habberstad AH, Sundstrøm S, et al. Prognostic value of histological features in diffuse astrocytomas WHO grade II. Int J Clin Exp Pathol 2012;5:152-8.

28. Foltyn M, Nieto Taborda KN, Neuberger U, et al. T2/ FLAIR-mismatch sign for noninvasive detection of IDHmutant $1 \mathrm{p} / 19 \mathrm{q}$ non-codeleted gliomas: validity and pathophysiology. Neurooncol Adv 2020;2:vdaa004.

29. Corell A, Ferreyra Vega S, Hoefling N, et al. The clinical significance of the T2-FLAIR mismatch sign in grade II and III gliomas: a population-based study. BMC Cancer 2020;20:450.

30. Goyal A, Yolcu YU, Goyal A, et al. The T2-FLAIRmismatch sign as an imaging biomarker for IDH and 1p/19q status in diffuse low-grade gliomas: a systematic review with a Bayesian approach to evaluation of diagnostic test performance. Neurosurg Focus 2019;47:E13.

31. Shaikh N, Brahmbhatt N, Kruser TJ, et al. Pleomorphic xanthoastrocytoma: a brief review. CNS Oncol 2019;8:CNS39.

32. Crespo-Rodríguez AM, Smirniotopoulos JG, Rushing EJ. MR and CT imaging of 24 pleomorphic xanthoastrocytomas (PXA) and a review of the literature. Neuroradiology 2007;49:307-15.

33. Yu S, He L, Zhuang X, Luo B. Pleomorphic xanthoastrocytoma: MR imaging findings in 19 patients. Acta Radiol 2011;52:223-8.

34. Marburger T, Prayson R. Angiocentric glioma: a clinicopathologic review of 5 tumors with identification of associated cortical dysplasia. Arch Pathol Lab Med 2011;135:1037-41.

35. Han G, Zhang J, Ma Y, et al. Clinical characteristics, treatment and prognosis of angiocentric glioma. Oncol Lett 2020;20:1641-8.

36. Bandopadhayay P, Ramkissoon LA, Jain P, et al. MYBQKI rearrangements in angiocentric glioma drive tumorigenicity through a tripartite mechanism. Nat Genet 2016;48:273-82. 
37. Qaddoumi I, Orisme W, Wen J, et al. Genetic alterations in uncommon low-grade neuroepithelial tumors: BRAF, FGFR1, and MYB mutations occur at high frequency and align with morphology. Acta Neuropathol 2016;131:833-45.

38. Shakur SF, McGirt MJ, Johnson MW, et al. Angiocentric glioma: a case series. J Neurosurg Pediatr 2009;3:197-202.

39. Preusser M, Hoischen A, Novak K, et al. Angiocentric glioma: report of clinico-pathologic and genetic findings in 8 cases. Am J Surg Pathol 2007;31:1709-8.

40. Ersen A, Canda MS, Men S, et al. Angiocentric Glioma: The Infiltrative Glioma with Ependymal Differentiation. Turk Patoloji Derg 2017;33:251-5.

41. Wood MD, Tihan T, Perry A, et al. Multimodal molecular analysis of astroblastoma enables reclassification of most cases into more specific molecular entities. Brain Pathol 2018;28:192-202.

42. Bell JW, Osborn AG, Salzman KL, et al. Neuroradiologic characteristics of astroblastoma. Neuroradiology 2007;49:203-9.

43. Regragui M, Karkouri M, Ibahioin K, et al. Astroblastoma: A rare glial tumor. Ann Pathol 2018;38:381-5.

44. Bondy ML, Scheurer ME, Malmer B, et al. Brain tumor epidemiology: consensus from the Brain Tumor Epidemiology Consortium. Cancer 2008;113:1953-68.

45. Fangusaro J. Pediatric high grade glioma: a review and update on tumor clinical characteristics and biology. Front Oncol 2012;2:105.

46. Schwartzentruber J, Korshunov A, Liu XY, et al. Driver mutations in histone $\mathrm{H} 3.3$ and chromatin remodelling genes in paediatric glioblastoma. Nature 2012;482:226-31.

47. Wu G, Diaz AK, Paugh BS, et al. The genomic landscape of diffuse intrinsic pontine glioma and pediatric nonbrainstem high-grade glioma. Nat Genet 2014;46:444-50.

48. Taylor KR, Mackay A, Truffaux N, et al. Recurrent activating ACVR1 mutations in diffuse intrinsic pontine glioma. Nat Genet 2014;46:457-61.

49. Fontebasso AM, Papillon-Cavanagh S, Schwartzentruber J, et al. Recurrent somatic mutations in ACVR1 in pediatric midline high-grade astrocytoma. Nat Genet 2014;46:462-6.

50. Buczkowicz P, Hoeman C, Rakopoulos P, et al. Genomic analysis of diffuse intrinsic pontine gliomas identifies three molecular subgroups and recurrent activating ACVR1 mutations. Nat Genet 2014;46:451-6.

51. Wang L, Li Z, Zhang M, et al. H3 K27M-mutant diffuse midline gliomas in different anatomical locations. Hum Pathol 2018;78:89-96.

52. Makepeace L, Scoggins M, Mitrea B, et al. MRI Patterns of Extrapontine Lesion Extension in Diffuse Intrinsic Pontine
Gliomas. AJNR Am J Neuroradiol 2020;41:323-30.

53. Aboian MS, Tong E, Solomon DA, et al. Diffusion Characteristics of Pediatric Diffuse Midline Gliomas with Histone H3-K27M Mutation Using Apparent Diffusion Coefficient Histogram Analysis. AJNR Am J Neuroradiol 2019;40:1804-10.

54. Castel D, Philippe C, Calmon R, et al. Histone H3F3A and HIST1H3B K27M mutations define two subgroups of diffuse intrinsic pontine gliomas with different prognosis and phenotypes. Acta Neuropathol 2015;130:815-27.

55. Aboian MS, Solomon DA, Felton E, et al. Imaging Characteristics of Pediatric Diffuse Midline Gliomas with Histone H3 K27M Mutation. AJNR Am J Neuroradiol 2017;38:795-800.

56. Korshunov A, Capper D, Reuss D, et al. Histologically distinct neuroepithelial tumors with histone 3 G34 mutation are molecularly similar and comprise a single nosologic entity. Acta Neuropathol 2016;131:137-46.

57. Mackay A, Burford A, Carvalho D, et al. Integrated Molecular Meta-Analysis of 1,000 Pediatric High-Grade and Diffuse Intrinsic Pontine Glioma. Cancer Cell 2017;32:520-37.e5.

58. Vettermann FJ, Felsberg J, Reifenberger G, et al. Characterization of Diffuse Gliomas With Histone H3G34 Mutation by MRI and Dynamic 18F-FET PET.Clin Nucl Med 2018;43:895-8.

59. Neumann JE, Dorostkar MM, Korshunov A, et al. Distinct Histomorphology in Molecular Subgroups of Glioblastomas in Young Patients. J Neuropathol Exp Neurol 2016;75:408-14.

60. Mondal G, Lee JC, Ravindranathan A, et al. Pediatric bithalamic gliomas have a distinct epigenetic signature and frequent EGFR exon 20 insertions resulting in potential sensitivity to targeted kinase inhibition. Acta Neuropathol 2020;139:1071-88.

61. Sturm D, Witt H, Hovestadt V, et al. Hotspot mutations in $\mathrm{H} 3 \mathrm{~F} 3 \mathrm{~A}$ and IDH1 define distinct epigenetic and biological subgroups of glioblastoma. Cancer Cell 2012;22:425-37.

62. Noushmehr H, Weisenberger DJ, Diefes K, et al. Identification of a $\mathrm{CpG}$ island methylator phenotype that defines a distinct subgroup of glioma. Cancer Cell 2010;17:510-22.

63. Sanson M, Marie Y, Paris S, et al. Isocitrate dehydrogenase 1 codon 132 mutation is an important prognostic biomarker in gliomas. J Clin Oncol 2009;27:4150-4.

64. Choi C, Ganji SK, DeBerardinis RJ, et al. 2-hydroxyglutarate detection by magnetic resonance spectroscopy in IDH-mutated patients with gliomas. Nat 
Med 2012;18:624-9.

65. Nicolaides TP, Li H, Solomon DA, et al. Targeted therapy for BRAFV600E malignant astrocytoma. Clin Cancer Res 2011;17:7595-604.

66. Giulioni M, Marucci G, Martinoni M, et al. Epilepsy associated tumors: Review article. World J Clin Cases 2014;2:623-41.

67. Shin JH, Lee HK, Khang SK, et al. Neuronal tumors of the central nervous system: radiologic findings and pathologic correlation. Radiographics 2002;22:1177-89.

68. Zanello $M$, Pages $M$, Tauziède-Espariat $A$, et al. Clinical, Imaging, Histopathological and Molecular Characterization of Anaplastic Ganglioglioma. J Neuropathol Exp Neurol 2016;75:971-80.

69. Zaky W, Patil SS, Park M, et al. Ganglioglioma in children and young adults: single institution experience and review of the literature. J Neurooncol 2018;139:739-47.

70. Koeller KK, Henry JM. From the archives of the AFIP: superficial gliomas: radiologic-pathologic correlation. Armed Forces Institute of Pathology. Radiographics 2001;21:1533-56.

71. Cheng CS, Ou CH, Chen JS, et al. Lhermitte-Duclos disease: A case report with radiologic-pathologic correlation. Radiol Case Rep 2019;14:734-9.

72. Casperson BK, Anaya-Baez V, Kirzinger SS, et al. Coexisting MS and Lhermitte-Duclos Disease. J Radiol Case Rep 2010;4:1-6.

73. Appay R, Pages M, Colin C, et al. Diffuse leptomeningeal glioneuronal tumor: a double misnomer? A report of two cases. Acta Neuropathol Commun 2020;8:95.

74. Cho HJ, Myung JK, Kim H, et al. Primary diffuse leptomeningeal glioneuronal tumors. Brain Tumor Pathol 2015;32:49-55.

75. Gardiman MP, Fassan M, Orvieto E, et al. Diffuse leptomeningeal glioneuronal tumors: a new entity? Brain Pathol 2010;20:361-6.

76. Lakhani DA, Mankad K, Chhabda S, et al. Diffuse Leptomeningeal Glioneuronal Tumor of Childhood. AJNR Am J Neuroradiol 2020;41:2155-9.

77. Dian S, Hermawan R, van Laarhoven A, et al. Brain MRI findings in relation to clinical characteristics and outcome of tuberculous meningitis. PLoS One 2020;15:e0241974.

78. Wang AC, Jones DTW, Abecassis IJ, et al. Desmoplastic Infantile Ganglioglioma/Astrocytoma (DIG/DIA) Are Distinct Entities with Frequent BRAFV600 Mutations. Mol Cancer Res 2018;16:1491-8.

79. Trehan G, Bruge H, Vinchon M, et al. MR imaging in the diagnosis of desmoplastic infantile tumor: retrospective study of six cases. AJNR Am J Neuroradiol 2004;25:1028-33.

80. Bader A, Heran M, Dunham C, et al. Radiological features of infantile glioblastoma and desmoplastic infantile tumors: British Columbia's Children's Hospital experience. J Neurosurg Pediatr 2015;16:119-25.

81. Bianchi F, Tamburrini G, Massimi L, et al. Supratentorial tumors typical of the infantile age: desmoplastic infantile ganglioglioma (DIG) and astrocytoma (DIA). A review. Childs Nerv Syst 2016;32:1833-8.

82. Takahashi A, Hong SC, Seo DW, et al. Frequent association of cortical dysplasia in dysembryoplastic neuroepithelial tumor treated by epilepsy surgery. Surg Neurol 2005;64:419-27.

83. Makola M, Cecil KM. Dysembryoplastic neuroepithelial tumor (DNET) and focal cortical dysplasia: Case report of two pediatric patients with imaging features. Inter J Diagnostic Imag 2017;4:31.

84. Luzzi S, Elia A, Del Maestro M, et al. Dysembryoplastic Neuroepithelial Tumors: What You Need to Know. World Neurosurg 2019;127:255-65.

85. Fernandez C, Girard N, Paz Paredes A, et al. The usefulness of MR imaging in the diagnosis of dysembryoplastic neuroepithelial tumor in children: a study of 14 cases. AJNR Am J Neuroradiol 2003;24:829-34.

86. Isler C, Erturk Cetin O, Ugurlar D, et al. Dysembryoplastic neuroepithelial tumours: clinical, radiological, pathological features and outcome. Br J Neurosurg 2018;32:436-41.

87. Zahra C, Grech R. Multinodular and Vacuolating Neuronal Tumor. Open Access Maced J Med Sci 2018;6:1697-8.

88. Hsu C, Kwan G, Lau Q, et al. Rosette-forming glioneuronal tumour: imaging features, histopathological correlation and a comprehensive review of literature. Br J Neurosurg 2012;26:668-73.

89. Gessi M, Moneim YA, Hammes J, et al. FGFR1 mutations in Rosette-forming glioneuronal tumors of the fourth ventricle. J Neuropathol Exp Neurol 2014;73:580-4.

90. Gao L, Han F, Jin Y, et al. Imaging features of rosetteforming glioneuronal tumours. Clin Radiol 2018;73:275-82.

91. Shih RY, Koeller KK. Embryonal Tumors of the Central Nervous System: From the Radiologic Pathology Archives. Radiographics 2018;38:525-41.

92. Cavalli FMG, Remke M, Rampasek L, et al. Intertumoral Heterogeneity within Medulloblastoma Subgroups. Cancer Cell 2017;31:737-54.e6.

93. Alves CAPF, Löbel U, Martin-Saavedra JS, et al. A 
Diagnostic Algorithm for Posterior Fossa Tumors in Children: A Validation Study. AJNR Am J Neuroradiol 2021. doi:10.3174/ajnr.A7057. [Epub ahead of print].

94. Perreault S, Ramaswamy V, Achrol AS, et al. MRI surrogates for molecular subgroups of medulloblastoma. AJNR Am J Neuroradiol 2014;35:1263-9.

95. Stock A, Mynarek M, Pietsch T, et al. Imaging Characteristics of Wingless Pathway Subgroup Medulloblastomas: Results from the German HIT/SIOPTrial Cohort. AJNR Am J Neuroradiol 2019;40:1811-7.

96. Blüml S, Margol AS, Sposto R, et al. Molecular subgroups of medulloblastoma identification using noninvasive magnetic resonance spectroscopy. Neuro Oncol 2016;18:126-31.

97. Lau CS, Mahendraraj K, Chamberlain RS. Atypical teratoid rhabdoid tumors: a population-based clinical outcomes study involving 174 patients from the Surveillance, Epidemiology, and End Results database (1973-2010). Cancer Manag Res 2015;7:301-9.

98. Hasselblatt M, Gesk S, Oyen F, et al. Nonsense mutation and inactivation of SMARCA4 (BRG1) in an atypical teratoid/rhabdoid tumor showing retained SMARCB1 (INI1) expression. Am J Surg Pathol 2011;35:933-5.

99. Wykoff CC, Lam BL, Brathwaite CD, et al. Atypical teratoid/rhabdoid tumor arising from the third cranial nerve. J Neuroophthalmol 2008;28:207-11.

100. Wesseling P. Embryonal tumor with multilayered rosettes (ETMR): signed, sealed, delivered .... Acta Neuropathol 2014;128:305-8.

101. Nowak J, Seidel C, Pietsch T, et al. Systematic comparison of MRI findings in pediatric ependymoblastoma with ependymoma and CNS primitive neuroectodermal tumor not otherwise specified. Neuro Oncol 2015;17:1157-65.

102.Pajtler KW, Witt H, Sill M, et al. Molecular Classification of Ependymal Tumors across All CNS Compartments, Histopathological Grades, and Age Groups. Cancer Cell 2015;27:728-43.

103. Mangalore S, Aryan S, Prasad C, et al. Imaging characteristics of supratentorial ependymomas: Study on a large single institutional cohort with histopathological correlation. Asian J Neurosurg 2015;10:276-81.

104. Yuh EL, Barkovich AJ, Gupta N. Imaging of ependymomas: MRI and CT. Childs Nerv Syst 2009;25:1203-13.

105.Pienkowska M, Choufani S, Turinsky AL, et al. DNA methylation signature is prognostic of choroid plexus tumor aggressiveness. Clin Epigenetics 2019;11:117. 106. Jaiswal AK, Jaiswal S, Sahu RN, et al. Choroid plexus papilloma in children: Diagnostic and surgical considerations. J Pediatr Neurosci 2009;4:10-6.

107. Lin H, Leng X, Qin CH, et al. Choroid plexus tumours on MRI: similarities and distinctions in different grades. Cancer Imaging 2019;19:17.

108. Smith AB, Smirniotopoulos JG, Horkanyne-Szakaly I. From the radiologic pathology archives: intraventricular neoplasms: radiologic-pathologic correlation.

Radiographics 2013;33:21-43.

109.Mufti ST, Jamal A. Primary intracranial germ cell tumors. Asian J Neurosurg 2012;7:197-202.

110. Packer RJ, Cohen BH, Cooney K. Intracranial germ cell tumors. Oncologist 2000;5:312-20. Erratum in: Oncologist 2000; 5 :following 438. Coney, K [corrected to Cooney, K].

111. Weiner HL, Finlay JL. Surgery in the management of primary intracranial germ cell tumors. Childs Nerv Syst 1999; 15:770-3.

112. Ogiwara H, Tsutsumi Y, Matsuoka K, et al. Apparent diffusion coefficient of intracranial germ cell tumors. J Neurooncol 2015;121:565-71.

113. Goldman S, Bouffet E, Fisher PG, et al. Phase II Trial Assessing the Ability of Neoadjuvant Chemotherapy With or Without Second-Look Surgery to Eliminate Measurable Disease for Nongerminomatous Germ Cell Tumors: A Children's Oncology Group Study. J Clin Oncol 2015;33:2464-71.

114. Morana G, Alves CA, Tortora D, et al. T2*-based MR imaging (gradient echo or susceptibility-weighted imaging) in midline and off-midline intracranial germ cell tumors: a pilot study. Neuroradiology 2018;60:89-99.

115.Echevarría ME, Fangusaro J, Goldman S. Pediatric central nervous system germ cell tumors: a review. Oncologist 2008;13:690-9.

116.Phi JH, Cho BK, Kim SK, et al. Germinomas in the basal ganglia: magnetic resonance imaging classification and the prognosis. J Neurooncol 2010;99:227-36.

117.Lee SM, Kim IO, Choi YH, et al. Early imaging findings in germ cell tumors arising from the basal ganglia. Pediatr Radiol 2016;46:719-26.

118. Minami N, Tanaka K, Kimura H, et al. Radiographic occult cerebellar germinoma presenting with progressive ataxia and cranial nerve palsy. BMC Neurol 2016;16:4.

119. Kalra SK, Vaid VK, Jaiswal AK, et al. Primary midbrain germinoma. J Clin Neurosci 2008;15:1282-4.

120. Goodwin TL, Sainani K, Fisher PG. Incidence patterns of central nervous system germ cell tumors: a SEER Study. J Pediatr Hematol Oncol 2009;31:541-4.

121.Zada G, Lopes MBS, Mukundan S Jr, et al. Germ Cell 
Tumors of the Sellar Region. In: Zada G, Lopes MBS, Mukundan S, et al. editors. Atlas of Sellar and Parasellar Lesions. Cham: Springer International Publishing, 2016:317-23.

122. Wang Y, Zou L, Gao B. Intracranial germinoma: clinical and MRI findings in 56 patients. Childs Nerv Syst 2010;26:1773-7.

123. Higano S, Takahashi S, Ishii K, et al. Germinoma originating in the basal ganglia and thalamus: MR and CT evaluation. AJNR Am J Neuroradiol 1994;15:1435-41.

124.Kakigi T, Okada T, Kanagaki M, et al. Quantitative imaging values of CT, MR, and FDG-PET to differentiate pineal parenchymal tumors and germinomas: are they useful? Neuroradiology 2014;56:297-303.

125.Löbel U, Rossi A. Pediatric Pineal Region Tumors. In: Rossi A, editor. Pediatric Neuroradiology. Berlin, Heidelberg: Springer, 2015:1-31.

126. Warren KE, Poussaint TY, Vezina G, et al. Challenges with defining response to antitumor agents in pediatric neuro-oncology: a report from the response assessment in pediatric neuro-oncology (RAPNO) working group. Pediatr Blood Cancer 2013;60:1397-401.

127. Fangusaro J, Witt O, Hernáiz Driever P, et al. Response assessment in paediatric low-grade glioma: recommendations from the Response Assessment in Pediatric Neuro-Oncology (RAPNO) working group. Lancet Oncol 2020;21:e305-16. Erratum in: Lancet Oncol 2020;21:e553.

128. Erker C, Tamrazi B, Poussaint TY, et al. Response assessment in paediatric high-grade glioma: recommendations from the Response Assessment in Pediatric Neuro-Oncology (RAPNO) working group. Lancet Oncol 2020;21:e317-29.

129. Cooney TM, Cohen KJ, Guimaraes CV, et al. Response

Cite this article as: Resende LL, Alves CAPF. Imaging of brain tumors in children: the basics-a narrative review. Transl Pediatr 2021;10(4):1138-1168. doi: 10.21037/tp-20-285 assessment in diffuse intrinsic pontine glioma: recommendations from the Response Assessment in Pediatric Neuro-Oncology (RAPNO) working group. Lancet Oncol 2020;21:e330-6.

130. Warren KE, Vezina G, Poussaint TY, et al. Response assessment in medulloblastoma and leptomeningeal seeding tumors: recommendations from the Response Assessment in Pediatric Neuro-Oncology committee. Neuro Oncol 2018;20:13-23.

131. Kessler AT, Bhatt AA. Brain tumour post-treatment imaging and treatment-related complications. Insights Imaging 2018;9:1057-75.

132. Morana G, Alves CA, Tortora D, et al. Added value of diffusion weighted imaging in pediatric central nervous system embryonal tumors surveillance. Oncotarget 2017;8:60401-13.

133. Guez D, Last D, Daniels D, et al. Radiation-induced vascular malformations in the brain, mimicking tumor in MRI-based treatment response assessment maps (TRAMs). Clin Transl Radiat Oncol 2018;15:1-6.

134.Louis DN, Wesseling P, Aldape K, et al. cIMPACTNOW update 6: new entity and diagnostic principle recommendations of the cIMPACT-Utrecht meeting on future CNS tumor classification and grading. Brain Pathol 2020;30:844-56.

135.Johnson DR, Giannini C, Jenkins RB, et al. Plenty of calcification: imaging characterization of polymorphous low-grade neuroepithelial tumor of the young. Neuroradiology 2019;61:1327-32.

136. Benson JC, Summerfield D, Carr C, et al. Polymorphous Low-Grade Neuroepithelial Tumor of the Young as a Partially Calcified Intra-Axial Mass in an Adult. AJNR Am J Neuroradiol 2020;41:573-8. 